Article

\title{
Improved MPPT Algorithm for Photovoltaic Systems Based on the Earthquake Optimization Algorithm
}

\author{
Efrain Mendez*(D), Alexandro Ortiz ${ }^{\mathbb{D}}$, Pedro Ponce ${ }^{\mathbb{D}}$, Israel Macias, David Balderas ${ }^{(\mathbb{D})}$ and \\ Arturo Molina \\ Tecnologico de Monterrey, School of Engineering and Sciences, Mexico City 14380, Mexico; \\ alexandro.ortiz@tec.mx (A.O.); pedro.ponce@tec.mx (P.P.); israel.macias@tec.mx (I.M.); \\ dc.balderassilva@tec.mx (D.B.); armolina@tec.mx (A.M.) \\ * Correspondence: efrain@tec.mx; Tel.: +52-5521-333-361
}

Received: 31 March 2020; Accepted: 16 May 2020; Published: 12 June 2020

check for updates

\begin{abstract}
Nowadays, owing to the growing interest in renewable energy, Photovoltaic systems (PV) are responsible of supplying more than $500,000 \mathrm{GW}$ of the electrical energy consumed around the world. Therefore, different converters topologies, control algorithms, and techniques have been studied and developed in order to maximize the energy harvested by PV sources. Maximum Power Point Tracking (MPPT) methods are usually employed with DC/DC converters, which together are responsible for varying the impedance at the output of photovoltaic arrays, leading to a change in the current and voltage supplied in order to achieve a dynamic optimization of the transferred energy. MPPT algorithms such as, Perturb and Observe (P\&O) guarantee correct tracking behavior with low calibration parameter dependence, but with a compromised relation between the settling time and steady-state oscillations, leading to a trade off between them. Nevertheless, proposed methods like Particle Swarm Optimization- (PSO) based techniques have improved the settling time with the addition of lower steady-state oscillations. Yet, such a proposal performance is highly susceptible and dependent to correct and precise parameter calibration, which may not always ensure the expected behavior. Therefore, this work presents a novel alternative for MPPT, based on the Earthquake Optimization Algorithm (EA) that enables a solution with an easy parameters calibration and an improved dynamic behavior. Hence, a boost converter case study is proposed to verify the suitability of the proposed technique through Simscape Power Systems ${ }^{\mathrm{TM}}$ simulations, regarding the dynamic model fidelity capabilities of the software. Results show that the proposed structure can easily be suited into different power applications. The proposed solution, reduced between $12 \%$ and $36 \%$ the energy wasted in the simulation compared to the $\mathrm{P} \& \mathrm{O}$ and $\mathrm{PSO}$ based proposals.
\end{abstract}

Keywords: optimization; nature-inspired; geo-inpired; boost converter; MPPT; EA; PV systems; energy harvesting; DC/DC converters

\section{Introduction}

In power electronics systems, energy generation through renewable sources has gained greater importance in an endless number of industrial and social applications. From among all the renewable energies, the Photovoltaic systems (PV) have gained great popularity for power supply solutions due its suitability around the world. Additionally, according to [1], energy harvested from PV systems are expected to be a great solution both for advanced economies and developing countries, since the energy demand is expected to grow $30 \%$ by 2040 (according to [2]).

In addition, ref. [2] points out that in order to move forward into a more sustainable future, a large expansion of renewable energy sources is required where Photovoltaic sources have a critical importance to achieve a better energetic transition from fossil fuels into renewable sources. 
Therefore, as explained by the International Energy Agency in [3], the PV energy provides the largest share of the expected global renewable capacity additions in most of the regions in the world. Thus, according to [4] the solar energy leads the renewable power capacity expansion, expected to be $50 \%$ between 2019 and 2024. Ref. [4] highlights that PV sources alone represent almost $60 \%$ of the expected total renewable energies growth, which can be translated into a $697 \mathrm{GW}$ capacity growth for solar energy sources.

Hence, the increasing need for cleaner energy leads to arising research topics, regarding converters topologies, control algorithms, and techniques required to optimize the energy harvested from PV sources. Therefore, among the different emerging topics, computational intelligence algorithms have gained popularity as alternatives for parameters identification, the dynamic optimization of energy harvested, and the control of different photovoltaic systems (as explained in [5]).

Consequently, the Maximum Power Point Tracking (MPPT) algorithms are widely studied, since they are responsible for tracking and acquiring the Maximum Power Point (MPP) of energy from the PV source. However, since the tracking of the MPP of a solar system is a dynamic optimization issue (as detailed later in this work), the problem requires a dynamic DC-optimizer (as explained in [6]). Henceforth, the MPPT methods are usually employed through DC/DC converters, which allows one to modify the impedance at the output of the PV source, achieving a modulation of the energy acquired in terms of the voltage $\left(V_{p v}\right)$ and current $\left(i_{p v}\right)$ from the PV array, by using the converter as a variable impedance source at the array terminals. In other words, together the MPPT algorithms with the DC/DC converters are responsible for varying the impedance at the output of photovoltaic arrays, leading to a change in the current and voltage they supply, in order to achieve a dynamic optimization of the transferred energy, as explained in [1].

On the one hand, the classic MPPT algorithms such as, the Perturb and Observe (P\&O), Hill-Climbing (HC), or Incremental Conductance (IC) algorithms have been widely selected for many PV systems, regarding their mathematical simplicity and easy implementation capabilities ([6]). Among them, the $\mathrm{P} \& \mathrm{O}$ has been the cornerstone for many solutions including IC and HC algorithms ([1]), where in spite of correct tracking behavior with low calibration parameter dependence, this type of algorithms based on the classic P\&O, carry with a high trade-off between the settling time and steady-state oscillations, due the magnitude of the perturbation that is continuously injected to the system, as explained in [7].

Thus, on the other hand, nature-inspired solutions have been developed addressing the issue where the classical Particle Swarm Optimization (PSO) metaheuristic algorithm has served as the basis of a different approach, where in [8] the PSO-based MPPT is presented as a solution with an improved settling time and with also lower steady-state oscillations, claiming the reduced steady-state oscillations as the main feature of the solution. Nevertheless, as studied by [9], the performance of PSO-based techniques are highly susceptible and dependent to a correct and precise parameter calibration, dramatically changing the searching behavior of the algorithm depending on the selected population size or the algorithm coefficients initialization (as detailed in [10]).

Henceforth, [6] explains that the parameters to be tuned in the PSO-based MPPT, the premature convergence problems, and even the complexity of the algorithm, have constrained the application of the algorithm into real PV systems. The premature convergence of the algorithm has also been studied in [11], where the results show how the classic PSO-based solution can fall into a local solution or induce greater oscillations due to the required re-initialization of the algorithm after irradiation changes, leading to conclude that the algorithm may not always ensure the expected tracking behavior (as validated in [12]).

Accordingly, this work presents a novel alternative for MPPT, based on the Earthquake Optimization Algorithm (EA), which enables an optimization with an easy parameters calibration and an improved dynamic behavior. The proposed algorithm is based on the main EA explained in [13], where the metaheuristic algorithm is characterized for using two different velocity 
equations, nature-inspired by the propagation equations of the $\mathrm{P}$ and $\mathrm{S}$ waves, which are part of physical earthquakes.

Therefore, the proposal seeks to take advantage of the performance of the fine search by the S-wave equation, in order to address the dynamic optimization issue of the MPPT for solar cells, allowing to transfer the fine search properties of the algorithm to this new solution, achieving a solution that improves energy harvesting. The searching behavior is always in the neighborhood of the best solution (as as explored in [14]), traduced into a metaheuristic search with an intermediate stage that allows the algorithm to always return to the global best.

Nonetheless, ref. [14] explores and compares many of the metaheuristic algorithms such as, PSO, Bat Algorithm (BA), Ant Colony (ACO), Grey Wolf Optimization (GWO), Genetic Algorithm, Cuckoo Search (CS), Artificial Bee Colony (ABC), and the EA among others. Thus, the comparison allows us to analyze how the fine search given by the S-wave velocity in the EA, makes it an efficient algorithm for the MPPT application, regarding the reduced searching space in terms of the control input that is given by the duty cycle.

Henceforth, in order to carry out the performance analysis of the proposed algorithm, a boost converter case study is proposed to verify the suitability of the proposed technique through Simscape Power Systems ${ }^{\mathrm{TM}}$ simulations in MATLAB $/$ Simulink ${ }^{\circledR}$, regarding the dynamic model fidelity capabilities of the software. Thereby, ref. [15] validates that simulations through Simscape ${ }^{\mathrm{TM}}$ can be used as valid testbeds for the performance of a plant. Accordingly, ref. [16] corroborates the capabilities of the Simscape Power Systems ${ }^{\mathrm{TM}}$ time-based simulations, for the study of the dynamic performance in control systems for power electronics. Thus, based on the study presented in [17], the efficiency of the power electronics system can be analyzed through the Simscape simulations.

Moreover, a boost converter topology was selected for the performed tests, since it has been widely used for PV applications as explored in [18,19], being that (according to [20]) it has a high voltage gain ratio, with simple implementation capabilities due the few elements required for its construction.

Nevertheless, as explained in [21], the presence of the non-minimum phase propitiated by the Right Half Plane Zero (RHPZ) in its transfer function, makes it a more challenging topology compared to buck converters or other topologies without RHPZ for MPPTs algorithms, since the algorithms could fall into erroneous behaviors due to the characteristic behavior of non-minimum phase elements, in this case since the voltage against each duty cycle change will first go in the opposite direction to the point at which it will stabilize (as explored by the resulsts presented in [22]), causing in turn erroneous readings that may cause confusion in control systems (as further explored in [23]).

Results show that the proposed technique can be easily suitable between different power applications, and as expected, conclude in a faster response compared to the $\mathrm{P} \& \mathrm{O}$ and the PSO-based MPPT algorithms. The quantification of the results showed that after the performed scenarios the EA-based MPPT consistently managed to harvest the most energy (measured through the power through the simulation time), with a lower standard deviation regarding the voltage at MPP, having been reduced between $12 \%$ and $36 \%$ of the energy wasted in the $10 \mathrm{~s}$ of simulations compared to both solutions.

The standard deviation in this work is used as a statistical tool in order to validate the behavior of the algorithms regarding steady-state oscillations, since by definition the standard deviation is the measurement of the amount of variation in a set of values. On the other hand, the statistical mean is estimated in order to evaluate the mean convergence error of the algorithms, which in addition to the standard deviation allows one to evaluate the behavior of the algorithms compared to the maximum achievable power.

This work presents in Section 2 the basic concepts of the mathematical model of the PV modules, later Section 3 details the dynamic optimization issue addressed through the MPPT algorithms. Additionally, Section 3 presents in Sections 3.1 and 3.2, the searching behavior of the P\&O and PSO-based MPPTs as a preamble to the main proposal of this work presented in Section 4. On the 
other hand, Section 5 explains the proposed case study scenarios, of which the results are broken down in Section 6. Finally, Section 7 shows the final conclusions of this work.

\section{Modeling of a PV Module}

In terms of energy harvesting sources, PV modules are responsible for converting sunlight to electrical energy, which according to [24], requires one to consider weather input data. Therefore, from the temperature and solar irradiation, current, voltage, and power harvested from the PV arrays can be estimated.

According to [1], the best way to analyze the dynamic behavior of a PV generator in simulated environments is adopting an equivalent model that enables the analysis of the key elements of the system, and therefore allowing to obtain its most relevant equations.

Hence, to understand how the input parameters affects the energy supplied by the PV modules, the single diode model with ohmic losses is chosen to study the dynamic optimization issue to be solved through the MPPT algorithms. The basic scheme of the electric diagram of the model is shown in Figure 1.

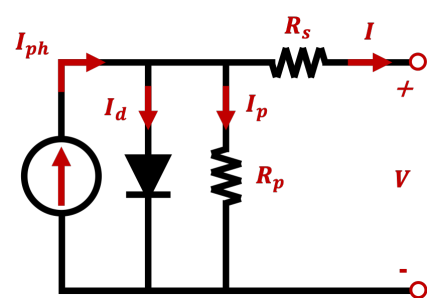

Figure 1. Single diode model with ohmic losses for Photovoltaic systems (PV) modules.

The model shown in Figure 1 corresponds to the single-diode model, which according to [25], may correctly model the performance for most type of solar cells, considering the performance at low irradiance for thin films-based solar cells as its main drawback. Nevertheless, the model is enough for this study purposes, knowing that the dynamic parameterization of the selected cells for the case study designs are the requirements needed to be fulfilled. In other words, the selected model is used to obtain the I-V (Current-Voltage) characteristics of the solar cells.

Then, from Figure 1, the output current from the solar cell (I) can be defined through Kirchhoff's current law, obtaining the expression shown by Equation (1).

$$
I=I_{p h}-I_{d}-I_{p}
$$

where $I_{p h}$ is the generated current from the PV cell, $I_{d}$ the current through the diode, and $I_{p}$ the current through the parallel resistor. Additionally, as described in [24], $I_{p h}$ can be rewritten in terms of the Irradiance $(G)$ and Temperature inputs $(T)$ giving rise to Equation (2).

$$
I_{p h}=\frac{G}{G_{S T C}}\left[I_{p h_{S T C}}+\mu_{s c}\left(T-T_{S T C}\right)\right]
$$

where $G$ stands for solar irradiation, $T$ is the cell temperature, $\mu_{s c}$ the coefficient of temperature of short circuit current, and the terms with subscript STC, represent the same variable but under Standard Test Conditions. On the other hand, Equation (3) represents the behavior of $I_{d}$ from Equation (1), in terms of $G$ and $T$.

$$
I_{d}=I_{0} \exp \left(\frac{V+I \cdot R_{s}}{a}-1\right)
$$

where $I_{0}$ is the reverse saturation current from the diode, $V$ the voltage supplied by the module, and $R_{S}$ the series resistance of the module shown in Figure 1. Additionally, $a$ is taken as a change of variable (also known as "the modified ideality factor" [24]), where the term is defined as: 


$$
a=\frac{N_{S} \cdot A \cdot k \cdot T_{C}}{q}=N_{S} \cdot A \cdot V_{T}
$$

where, $V_{T}$ is known as the thermal voltage, according to $[1,25,26], A$ is the ideality factor of the cell, $N_{s}$ the number series connected cells in the module, $k$ the Boltzmann constant $1.381 \times 10^{-23} \mathrm{~J} / \mathrm{K}$, $q$ the electron charge factor (constant $1.602 \times 10^{-19} \mathrm{C}$ ), and $T_{c}$ the temperature in the cell. Additionally for Equation (3), $I_{0}$ can be defined as [24]:

$$
I_{0}=\left[I_{\text {SC }_{S T C}} \exp \left(\frac{-V_{o c}}{a}\right)\right]\left(\frac{T_{\mathcal{C}}}{T_{\mathcal{C}_{S T C}}}\right)^{3} \exp \left[\left(\frac{q \varepsilon_{G}}{A K}\right)\left(\frac{1}{T_{\mathcal{C S T C}_{S T}}}-\frac{1}{T_{\mathcal{C}}}\right)\right]
$$

where $I_{s c}$ is the short circuit current, $V_{o c}$ the voltage in open circuit, and $\varepsilon_{G}$ the material's band gap energy. Finally from Equation (1), $I_{p}$ is the current leak through the parallel resistor and therefore according to [24] $I_{p}$ can be taken as:

$$
I_{p}=\frac{V+R_{s} \cdot I}{R_{p}}
$$

Henceforth, as explained in [24,25], the Voltage against Current characteristic profile can be obtained by sweeping voltages in order to estimate the current in every point, meanwhile the Voltage against Power profile can be obtained by the existing relation $P=V \cdot I$.

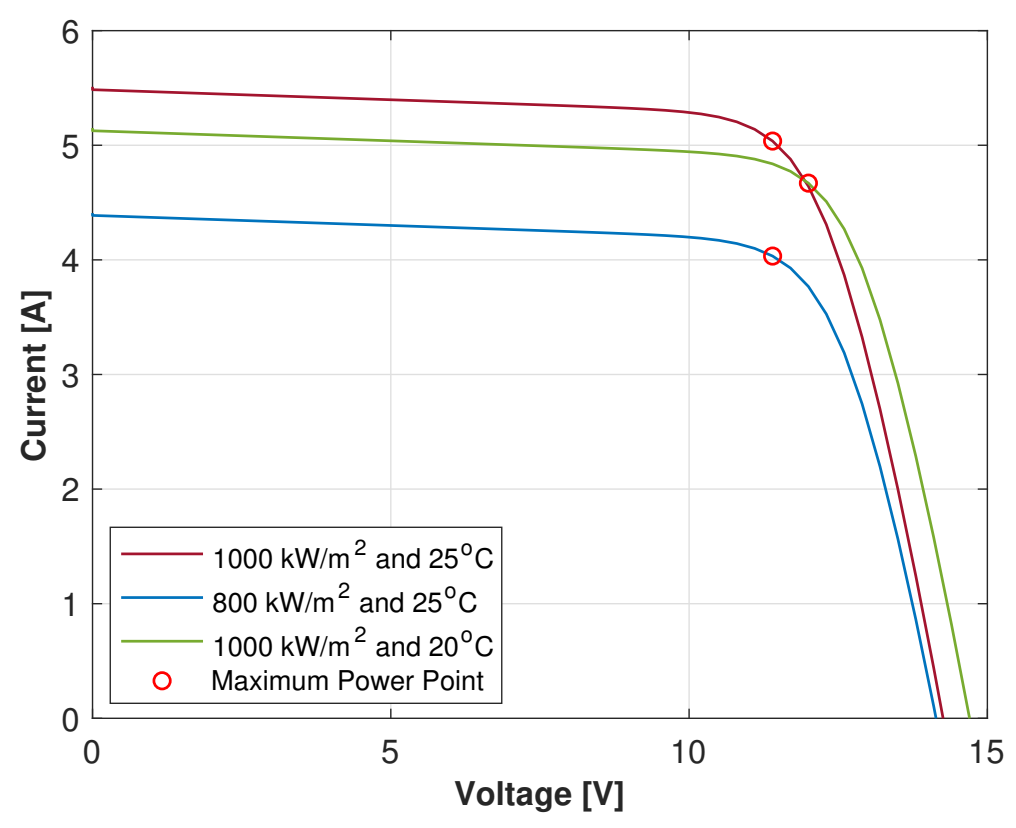

Figure 2. Current against Voltage profiles, for different Irradiances and Temperatures input values.

Then, Figure 2 shows the I-V characteristics of the solar modules selected for the low power simulations in this work, where the MPP is highlighted in the curves, exemplifying the variations of the MPP regarding the irradiance or temperature changes.

Meanwhile, the curves analyzed in Figure 2 can be processed in order to obtain the power against voltage features, a profile that is shown in Figure 3 where it can be seen how the maximum achievable power may be affected by the input changes regarding to the unpredictable weather changes. The above leads to the dynamic optimization issue to be solved by the MPPT algorithms. 


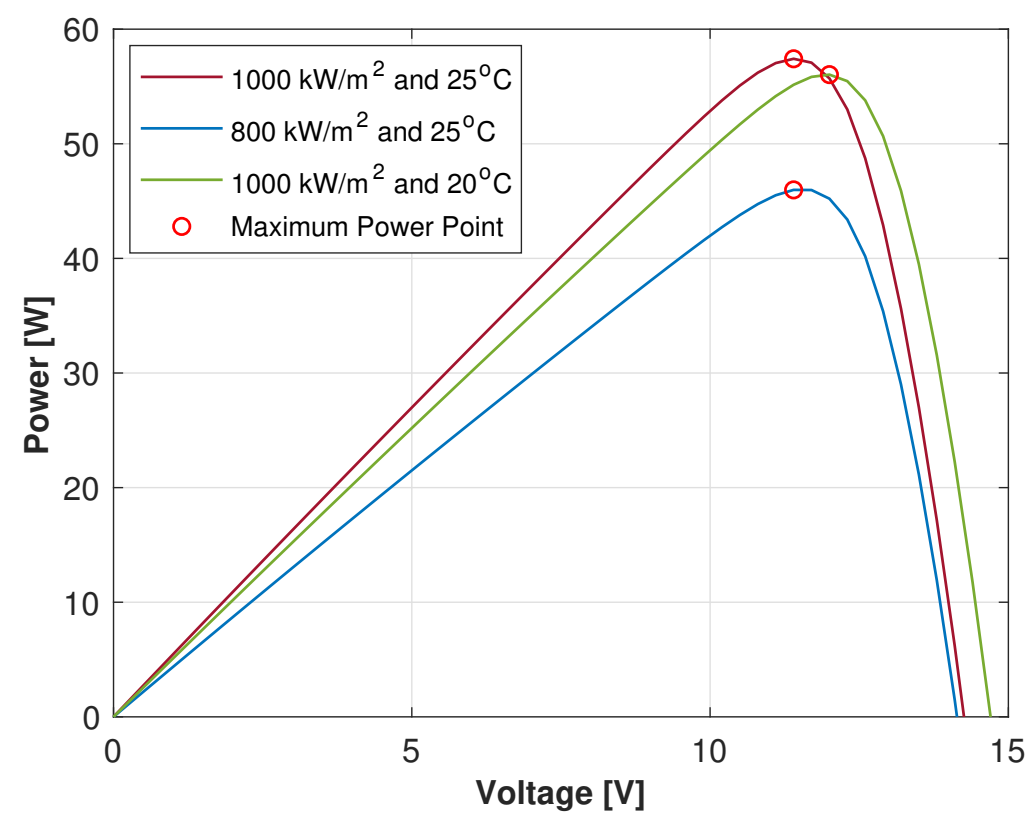

Figure 3. Power against Voltage profiles, for different Irradiances and Temperatures input values.

\section{Maximum Power Point Tracking}

Assuming the Maximum achievable power as a dynamic point with unpredictable variations due to the Temperature and Irradiation changes, the issue has to be dealt by a dynamic optimizator. As explained in [1], DC/DC converters can achieve a dynamic optimization of the impedance of the circuit, in order to control the transferred power through the regulation of the duty cycle $d$ of the converter. Therefore, the control parameter of the system is taken as $d$ of the main switch or switches of the converter (depending on the selected topology for the application).

In [23], the conversion ratio of different converter topologies regarding the duty cycle are explained, and in [1] it is explained how the conversion ratio can be explained as the equivalent resistance and voltage at the PV source terminals.

Therefore, to determine the path the duty cycle has go through, in order to ensure a Maximum Power Point operation of the PV source regardless the irradiance and temperature conditions, Maximum Power Point Tracking (MPPT) controllers are implemented. Figure 4 shows how the maximum power point moves depending on different variations, exemplifying the behavior first in STC (red profiles), then with a $20 \%$ of irradiance reduction (blue curves), next to a $20 \%$ of temperature reduction (green profiles), and finally with a $20 \%$ or reduction in both irradiation and temperature input conditions (purple profiles). In the case of all curves from Figure 4, the profiles with continuous lines are current curves, which are plotted in respect to the the left axis. Meanwhile the curves with segmented lines are plotted in respect to the right axis, representing the power behavior resulted from the Current against Voltage curves.

According to [9], MPPT controllers base their operation on data acquisition regarding the instantaneous current and voltage values sensed by the PV generator terminals. Moreover, by the knowledge of the obtained power related to the actual duty cycle in the converter, the MPPT takes the acquired data as comparison points in order to trace a route to be followed by the new duty cycles, depending on the tracking algorithm implemented. 


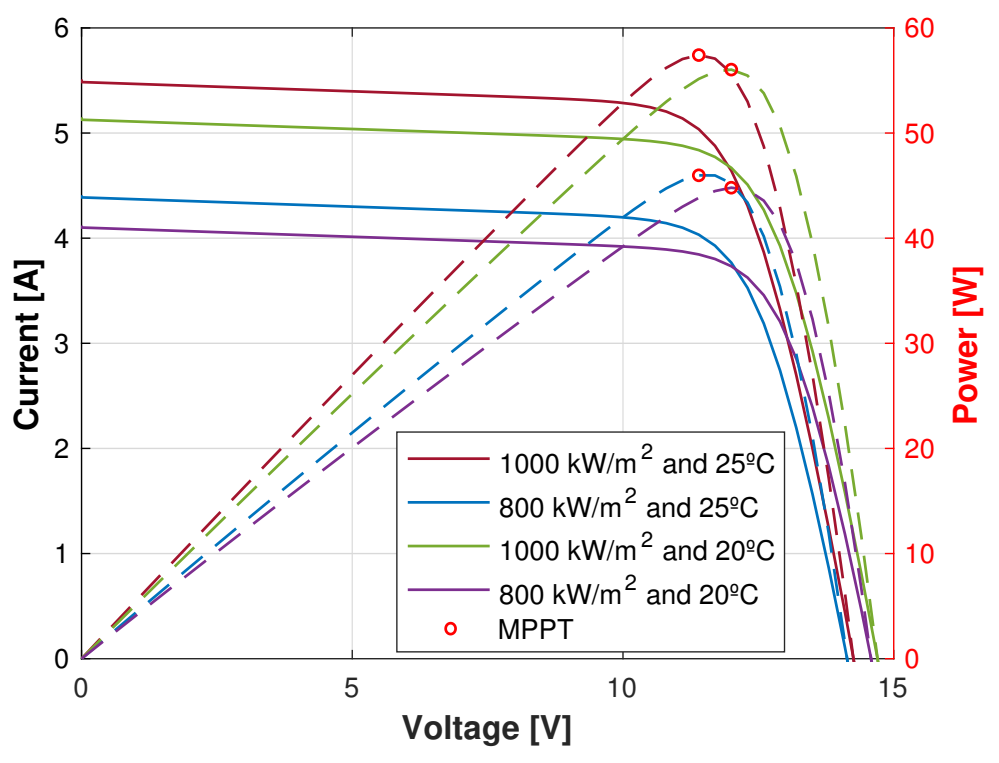

Figure 4. Current (left axis) and Power (right axis) against Voltage profiles, for different Irradiances and Temperatures input values.

\subsection{Perturb and Observe}

As explained earlier, as the duty cycle is the control parameter to be moduled in order to achieve a correct power tracking, the dynamic optimization issue can be dealed by different MPPT solutions, where one of the most implemented algorithms is the classic Perturb and Observe (P\&O) algorithm that, as studied in $[1,9,27]$, has been widely implemented and served for inspiration of many other algorithms due to its simplicity and effectiveness relation.

Basically, the $\mathrm{P} \& \mathrm{O}$ algorithm bases its operation on the injection of small perturbations into the system, in other words small variations of duty cycle, whose effects are measured in order to evaluate if the response is better or worse than the obtained from the previous duty cycle, therefore the result traces the road to be followed in the direction of the MPP.

Hence, to better explain the operation of the algorithm, Figure 5 shows a basic flow chart of the $\mathrm{P} \& \mathrm{O}$ MPPT. The algorithm starts with the system in a determined operating point, the initial point is usually set with the previous knowledge of the design parameters of the system, which means that it can be initialized with the estimated duty cycle operating in STC.

The actual state of the systems is perturbed every $T_{P}$ period of time, by changing the duty cycle $d$ in order to apply a voltage variation to the PV source terminals. After each applied disturbance, the algorithm seeks to compare the resultant power supplied by the PV array $\left(P_{P V}\right)$. The change in the actual measured Power $\left(P_{P V_{1}}\right)$, is compared to its previous power value $\left(P_{P V_{0}}\right)$, leading to the direction of the next perturbation to be applied.

It is important to highlight that, in order to achieve a good measure of the power after the perturbation, $T_{P}$ must be selected as a waiting time so that the system can stabilize since the presence of non-minimum phases in some converter topologies or even the same response speed of the system can be impediments to properly measure whether the response was better or earlier after the duty applied changes. 


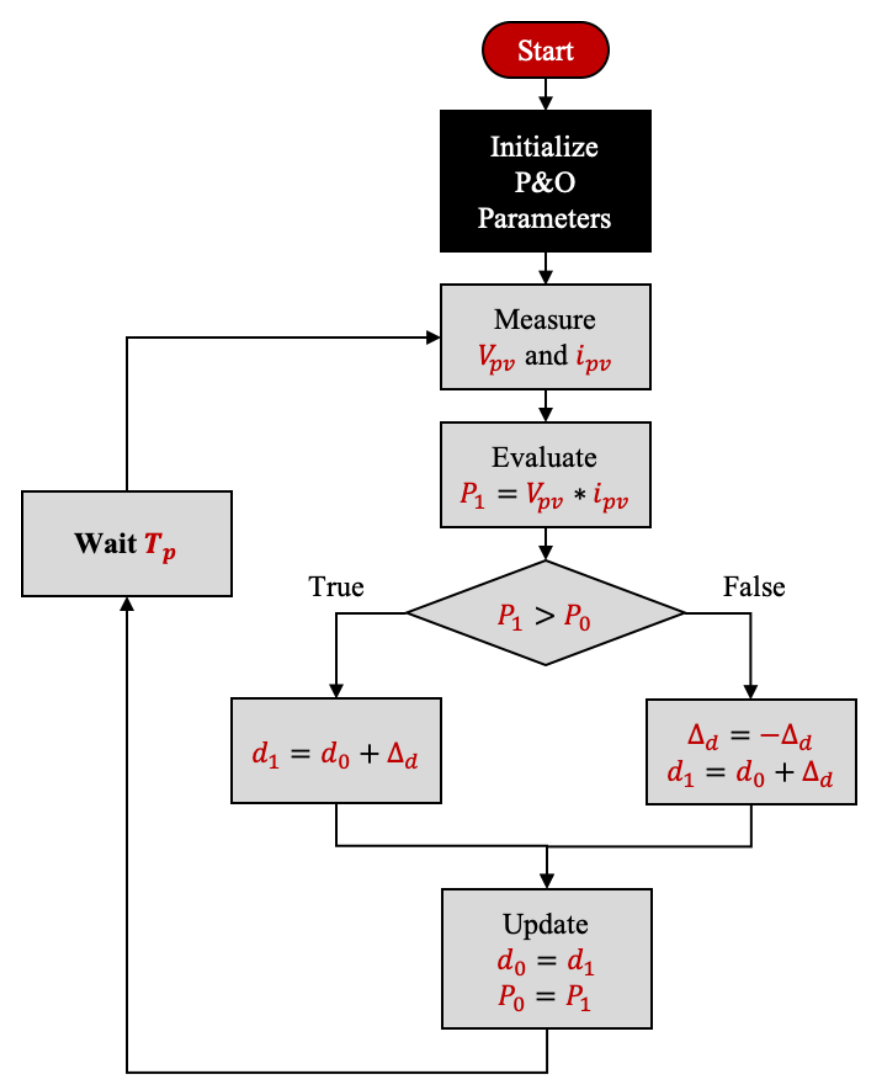

Figure 5. Flow chart of the Perturb and Observe (P\&O) Maximum Power Point Tracking (MPPT) algorithm.

\subsection{Particle Swarm Optimization Based Algorithm}

As explained in [1], the P\&O MPPT algorithm has served as inspiration for plenty of MPPT applications, within which, applications based on metaheuristic algorithms are found. Thus, a MPPT method based on the Particle Swarm Optimization (PSO) algorithm has been proposed by [8], where the online optimization is performed by perturbations applied through the classical PSO's velocity and position equations.

Firstly, the seminal version of the metaheuristic algorithm was introduced in [28], where the original PSO operates via a set of particles moving towards the personal and global best solutions from a set of particles, iterating until the expected error or maximum iterations conditions are fulfilled. Nevertheless, the most implemented version of the PSO algorithm contemplates an inertial weight coefficient, as described in [29,30]. Therefore, equations used to update each particle's velocity $(v)$ and position $(x)$ are given by:

$$
\begin{gathered}
v_{i}^{n+1}=w \cdot v_{i}^{n}+c_{1} \cdot r_{1} \cdot\left(x_{\text {best }}^{n}-x_{i}^{n}\right)+c_{2} \cdot r_{2} \cdot\left(G_{\text {best }}^{n}-x_{i}^{n}\right) \\
x_{i}^{n+1}=x_{i}^{n}+v_{i}^{n}
\end{gathered}
$$

where $C_{1}$ and $C_{2}$ are the acceleration constants and $w$ is the inertia weight that allows the balance of the global and local search capabilities, the coefficient should be normally calibrated between 0 and 1 as recommended in [31], in order to guarantee convergence. On the other hand, $r_{1}$ and $r_{2}$ in Equation (7), are random variables calculated between 0 and 1 for every particle on every iteration [28].

In the case of the portability of the algorithm for its application as a MPPT tool, the positions given by $x$ are taken as the duty cycles to be uploaded, meanwhile the velocities $(v)$ are the variations to be uploaded into the $j$ position, so that the behavior inspired from the P\&O algorithm is achieved. Therefore, Figure 6 shows the flowchart of the PSO-based MPPT algorithm, where $G_{b e s t}$ and $x_{\text {best }}$ are the best global and personal solutions of the particles. Additionally, it is important to highlight that 
MPPT approaches based on metaheuristic methods, retain most of the virtues and hence the defects of the original metaheuristic optimization algorithms, as analyzed in $[8,9]$.

Thereafter, Figure 6 shows the general structure of the PSO adapted as a MPPT algorithm, where it can be analyzed that after the initialization of the PSO parameters, the algorithm begins with the measurement of the voltage and the current from the PV source, then the process has two threads depending on whether the algorithm is loading the particles to the duty cycle or not. When the counter of particles $j$ differs from the number of particles $N_{P}$, the positions of each particle is charged one by one to the duty cycle, just as in the $\mathrm{P} \& \mathrm{O}$ algorithm, perturbing the system and observing the results.

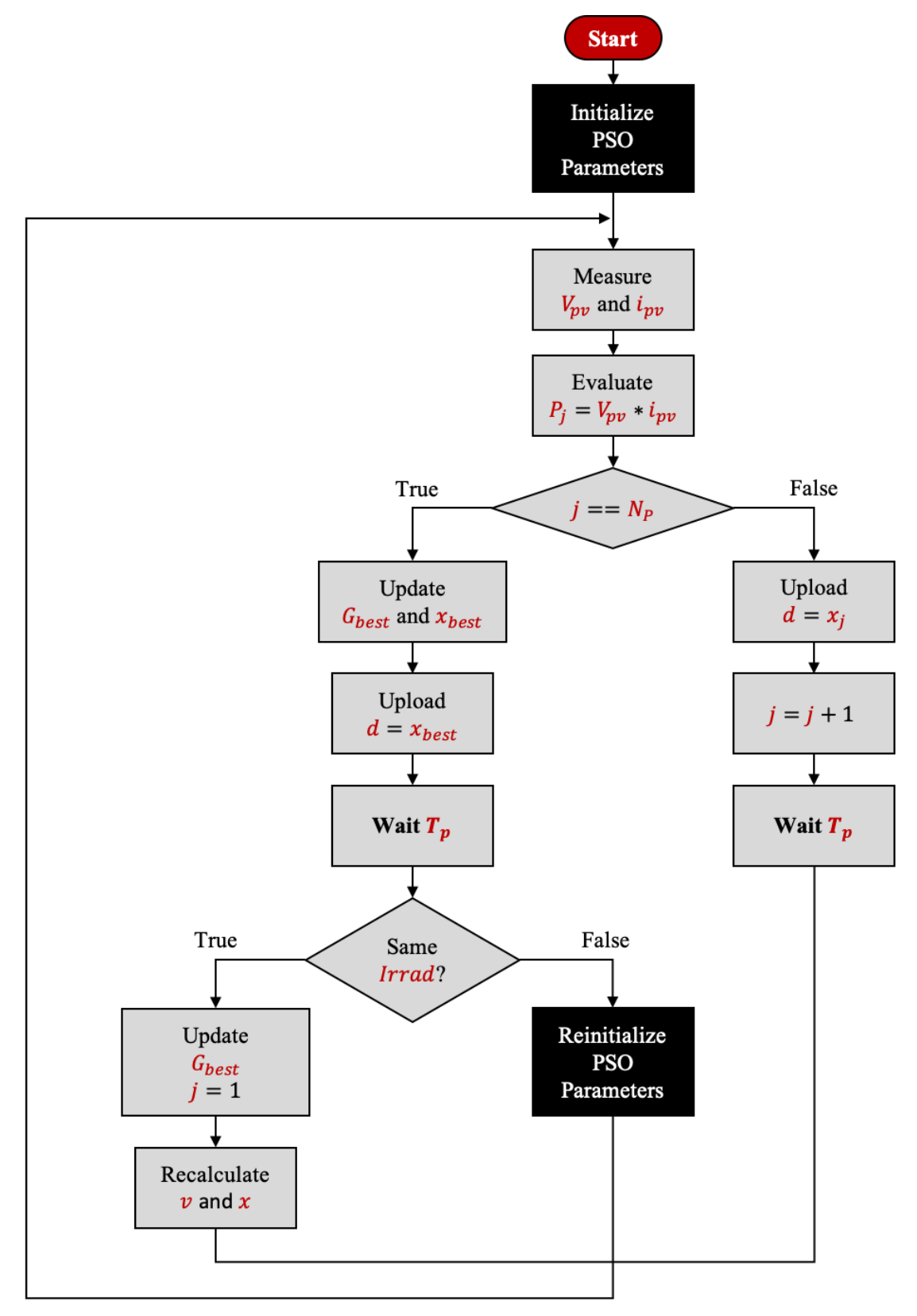

Figure 6. Flow chart of the Particle Swarm Optimization (PSO) MPPT algorithm.

On the other hand, when $j==N_{P}$, the method takes the natural behavior of the typical PSO, by obtaining and updating the MPP value and the best duty cycle positions. Additionally as explained in [9], a scanning process has to be made in order to detect irradiation changes, a task that can be achieved by comparing the previous MPP value against the new one, determining if the system still achieves the saved maximum power transfer with the duty cycle saved as the current best position. 
If a change in solar irradiation is detected, then the PSO parameters must be reinitialized to ensure that the algorithm does not stagnate in a local solution.

\section{Proposed MPPT Based on the Eartquake Algorithm}

The Earthquake Algorithm (EA) is the first geo-inspired metaheuristic algorithm (as explained in [14]), based on the behavior of the $P$ and $S$ waves existing in earthquakes. The first sketch of the algorithm appeared in [32], while the first complete version of the algorithm was formally introduced in [33], with its extended version in [13].

Therefore, as explained in [13], the algorithm takes advantage of the characteristics between wave velocities, where the $P$-wave is faster on the earth material compressibility, meanwhile the $S$-wave is slower and directly depends on rocks elasticity causing epicenters to move up and down, constantly perpendicular to the wave direction. For that reason (also from [13]), Equations (9) and (10), are the given mathematical expressions for the $P$ and $S$ waves velocities respectively.

$$
\begin{gathered}
v_{p}=\sqrt{\frac{\lambda+2 \mu}{\rho}} \\
v_{s}=\sqrt{\frac{\mu}{\rho}}
\end{gathered}
$$

where $v_{p}$ and $v_{s}$ are the waves velocities, $\lambda$ and $\mu$ the Lamé parameters, and $\rho$ the density of material. As explained in $[13,33]$, the optimal relation for the Lamé parameters was found to be 1.5 , consequently:

$$
\lambda=\mu=1.5 \mathrm{GPa} .
$$

Besides, for the implementation of the classic version of the algorithm, the density of the solids $(\rho)$ is used as a random value, selected from a range between 2200 and $3300 \mathrm{~kg} / \mathrm{m}^{3}$, also according to [13]. Nevertheless, in order to determine whether to use $v_{p}$ or $v_{s}$, for the algorithm it is a key element to define an operation range for the $S$-wave, which will be referred in [13] as the S-range or $S r$.

However, the main structure of the adaptation of the geo-inspired algorithm for the MPPT implementation is shown in Figure 7. Where, as explained in [13], the EA within its S-range (Sr) has a fine searching behavior, characterized for "orbiting" the global best value. Thus, extrapolating that feature for the dynamic optimization of the MPP tracker, the solution requires to contemplate that the epicenters should be "orbiting" around the MPP, to ensure a correct optimizing behavior.

Under those circumstances, a searching flag $\left(S_{\text {flag }}\right)$ is implemented to insert the best duty cycle within the searching positions, achieving the expected route-ways during the searching stage, and also extending the time that the system will remain in the considered MPP.

Unlike the PSO approach, the original EA has two velocity equations, which is why for this first adaptation of the algorithm, it is important to highlight that the $v_{S}$ equation is taken for this first approach of the algorithm adaptation.

Moreover, in order to clarify the similarities and differences between the original EA and the EA-based MPPT, the main features of this algorithm are enlisted below. The EA-based MPPT algorithm proposed in this paper takes advantage of the finer search of the original EA given by Equation (10), which allows dealing with the dynamic optimization, where the inputs of the EA-MPPT system are $V_{p} v$ and $I_{p} v$ leading to the fine optimization of the duty cycle (control parameter) for the power converter. Figure 8 highlights the main parameters of the system, where the inputs and outputs of the MPPT are summarized through the MPPT block.

Nevertheless, the main differences between this proposal and the original EA are based on the suitability requirements of PV systems, since this algorithm returns to the global best duty cycle after evaluating an epicenter and allows verifying how the irradiation features are changing before uploading the next epicenter, which in addition performs a faster reaction against irradiation changes 
compared to other algorithms, since algorithms like PSO-based MPPT monitors the irradiation changes after all sets of particles are induced into the system (as explored through the differences between Figures 6 and 7).

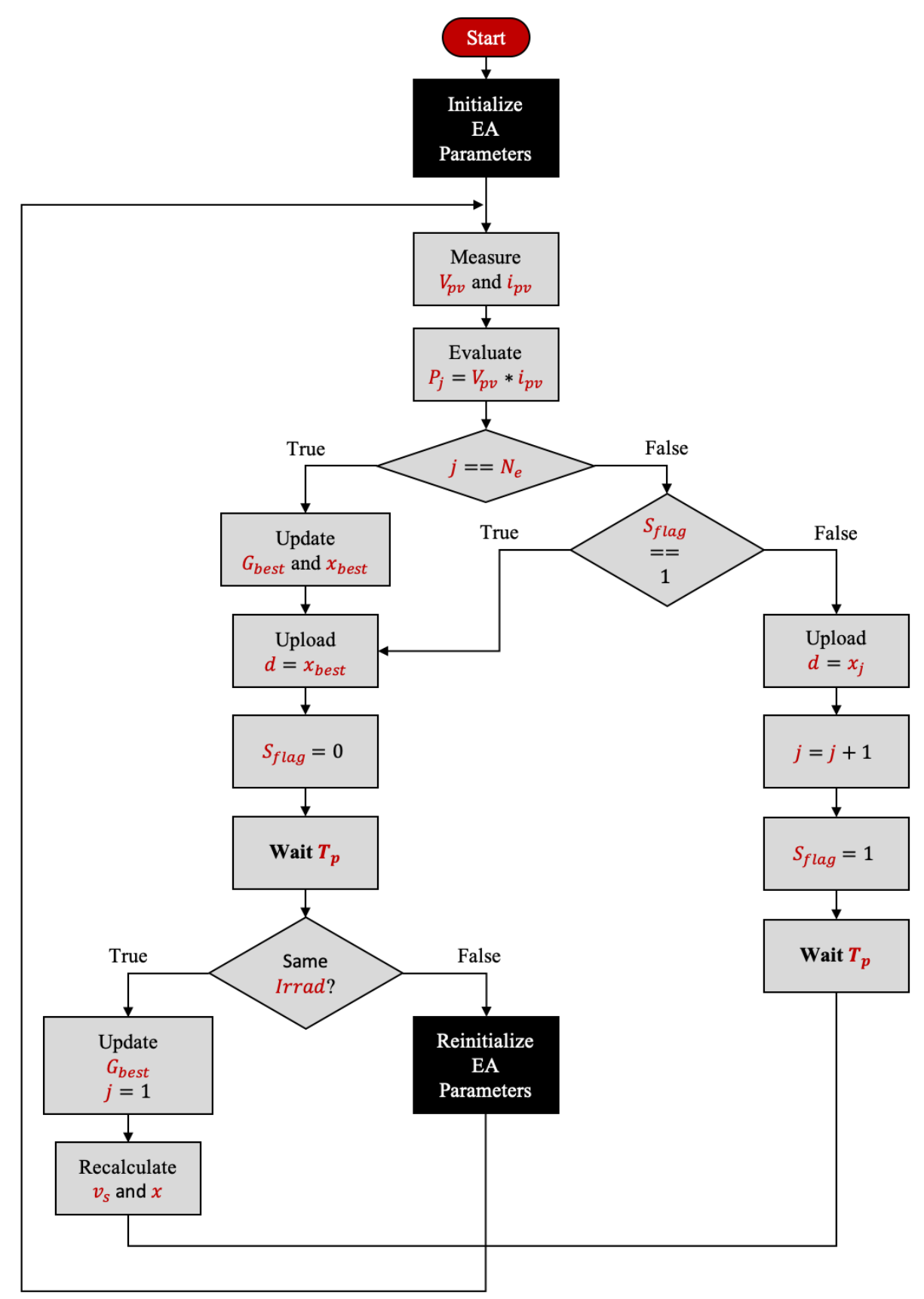

Figure 7. Flow chart of the Earthquake Optimization Algorithm (EA) MPPT algorithm.

\section{Case Study}

In order to compare the performance of the MPPT algorithms, two case studies are presented using the circuit topology shown in Figure 8. The first case study takes a low power generation application, meanwhile the second one takes an application in the order of [kW] as a case study. 


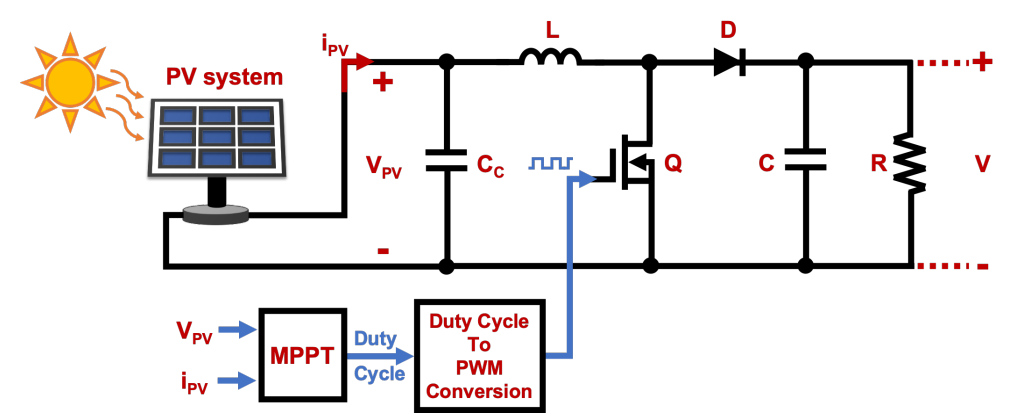

Figure 8. Selected topology for the case study.

\subsection{DC/DC Converter}

On the one hand, the designed designed boost converter for the low power simulations was designed to operate in CCM (Continuous Conduction Mode) for a typical $12 \mathrm{~V}$ input voltage conversion into $24 \mathrm{~V}$ output voltage at $2 \mathrm{~A}$. A summary of the design boost converter is shown in Table 1 where the nominal values of operation, such as the switching frequency, input and output voltages, the output power and current are presented.

Additionally, the boost converter for the high power simulations was based on the design studied in [34], also operating in CCM but now for a $300 \mathrm{~V}$ input voltage conversion into $600 \mathrm{~V}$ output voltage at $5.18 \mathrm{~A}$. The switching frequency, input and output voltages, the output power, and current are summarized in Table 2.

Table 1. Low power converter parameters.

\begin{tabular}{cc}
\hline Input voltage & $12 \mathrm{~V}$ \\
Switching frequency & $120 \mathrm{kHz}$ \\
Duty cycle & $50 \%$ \\
Inductance & $47 \mathrm{mH}$ \\
Capacitance & $220 \mu \mathrm{F}$ \\
Load resistance & $12 \Omega$ \\
Output power & $48 \mathrm{~W}$ \\
Output voltage & $24 \mathrm{~V}$ \\
Output current & $2 \mathrm{~A}$ \\
\hline
\end{tabular}

Table 2. High power converter parameters.

\begin{tabular}{cc}
\hline Input Voltage & $300 \mathrm{~V}$ \\
Switching frequency & $10 \mathrm{kHz}$ \\
Duty Cycle & $50 \%$ \\
Inductance & $280 \mathrm{mH}$ \\
Capacitance & $38.3 \mu \mathrm{F}$ \\
Load resistance & $115.84 \Omega$ \\
Output power & $3.108 \mathrm{~kW}$ \\
Output voltage & $600 \mathrm{~V}$ \\
Output current & $5.18 \mathrm{~A}$ \\
\hline
\end{tabular}

Moreover, Figure 9 shows the dynamic behavior of the converter from Table 1, where the different profiles were obtained through the step response of the converter against different duty cycles. Furthermore, using a similar duty cycle sweep as for the low power converter, it was possible to obtain the power profiles for the converter from Table 2, where the curves are shown in Figure 10. In both Figures 9 and 10, the nominal power profiles are highlighted as red curves. 


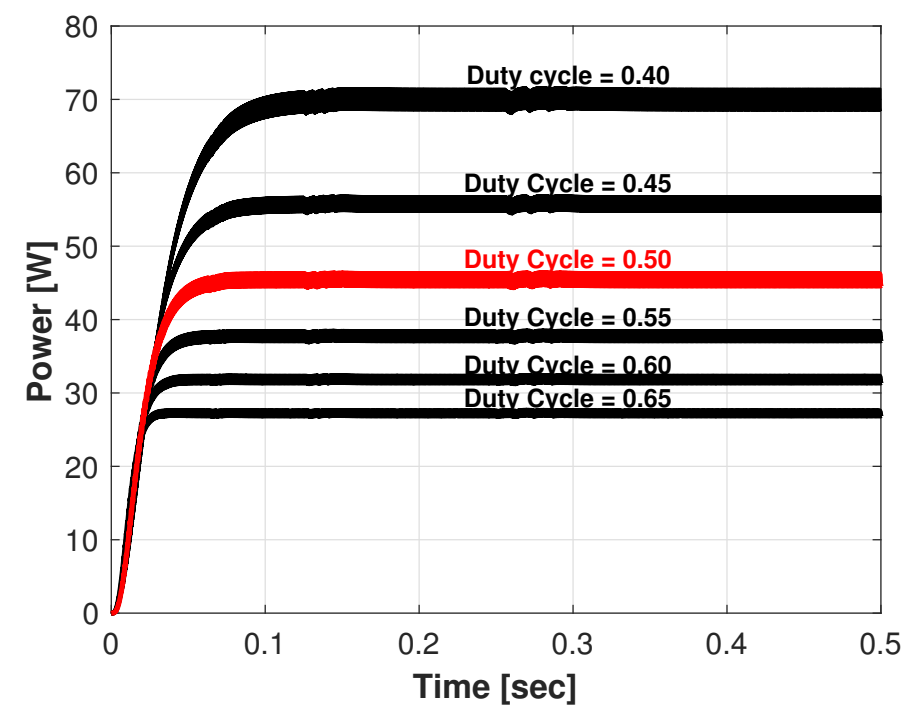

Figure 9. Power against time profiles with different duty cyle values, for the converter in Table 1.

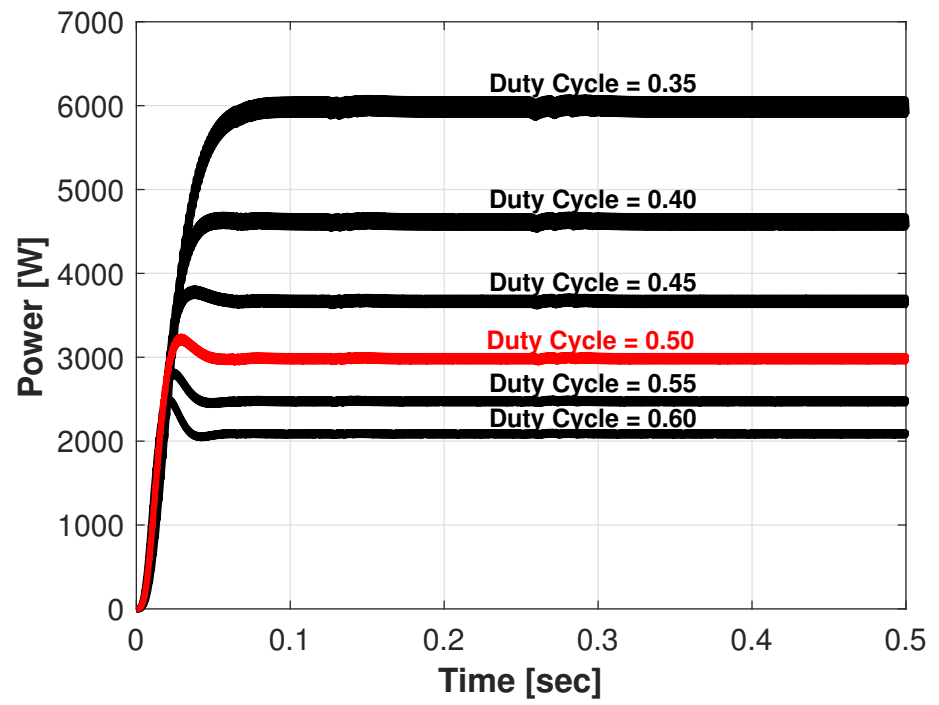

Figure 10. Power against time profiles with different duty cycle values, for the converter in Table 2.

Nonetheless, many simulations regarding MPPT algorithms have been performed using boost converter topologies. Nevertheless, some of them do not consider the changes in the dynamic behavior of the converter due to the input capacitor (coupling capacitor). Still, it is important to highlight that different coupling capacitors may affect the transient response of the converter (as explored in [35]).

Therefore, in order to ensure the fidelity of the results from the simulations, all the performed tests include the input capacitance of the system. In this case, the coupling capacitor $\left(C_{c}\right.$ as shown in Figure 8) used for the interconnection with the PV arrays, for both converter cases was selected as a $1000 \mu \mathrm{F}$ capacitance. The correct selection of this capacitance allows a correct dynamic response of the when the input power changes in time, reducing the undershoot when the power goes down and reducing the overshoots when the input steps up.

\subsection{PV Arrays}

On the other hand, in order to give a wider performance response analysis, two different profiles were used to simulate different irradiance inputs on the PV arrays. For the low power simulations, 
Figure 11 shows the used irradiation profile and Figure 12 shows the profile implemented for high power simulation.

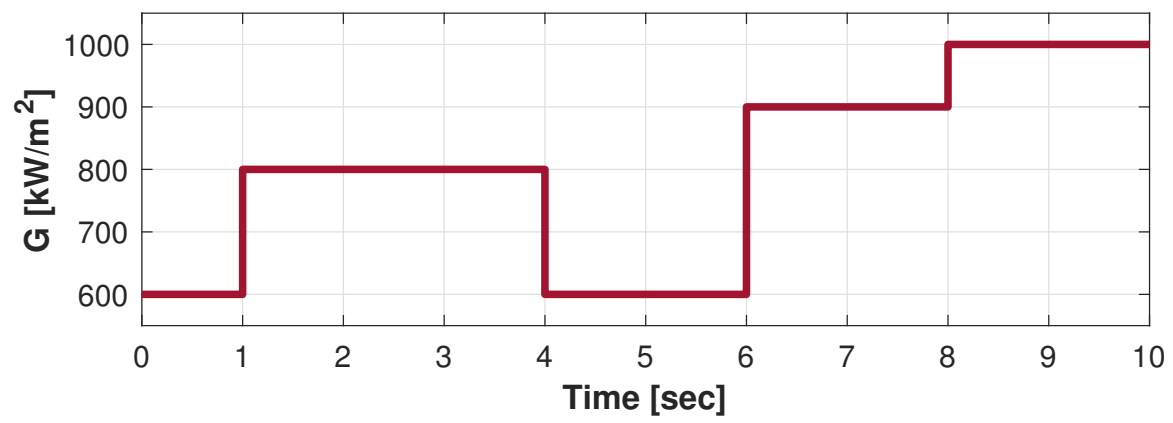

Figure 11. Irradiance profile used for low power simulations.

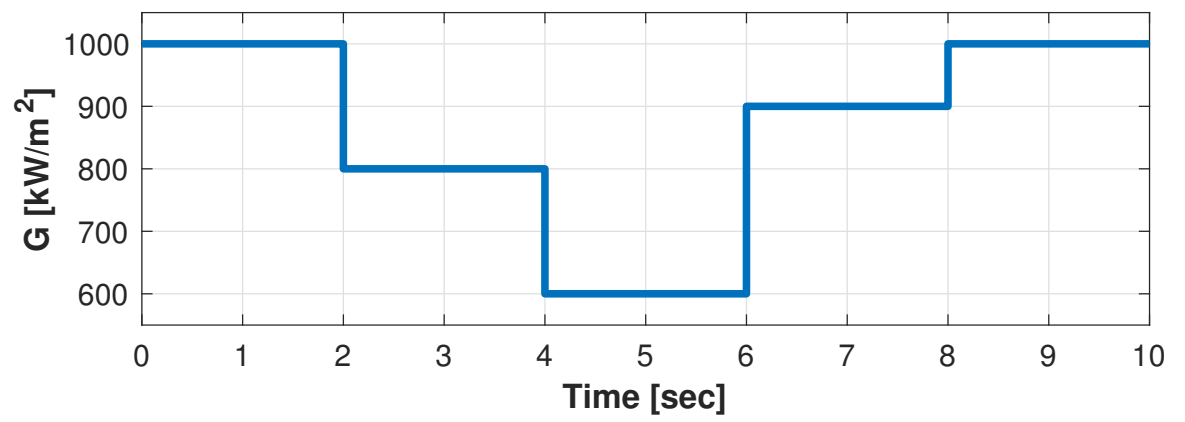

Figure 12. Irradiance profile used for higher power simulations.

In the case of the Figure 11 irradiation profile, it was designed in order to evaluate the response of the algorithms against different increments, regarding the irradiation through time. Therefore, the profile allows one to analyze the convergence velocity of the algorithms, where the speed of the algorithms to find the MPP will be evident, starting from a lower irradiance and seeking a better use of the time with greater solar energy.

Now, in the case of the irradiation profile shown in Figure 12, the irradiation changes were designed with the objective of testing the performance of the algorithms against different aggressive up/down step changes. Hence, for this proposed test, the converter designed for higher power is taken for the task, regarding the fact that at a higher power conversion the duty cycle changes have a greater effect in the system, maximizing also the effects of the error.

Moreover, knowing that the main objective is to evaluate the dynamic performance of the algorithms compared in this work, in every simulation case the temperature remained as a fixed variable at $25^{\circ} \mathrm{C}$, testing then the performance of the system when the irradiation dynamically steps up or down.

Nevertheless, it is important to highlight that in spite of the fact that the aggressive irradiation changes are not feasible in reality (at least as stated in Figures 11 and 12), the units of time were taken that way in order to analyze and validate the potential of each optimization method. The parameters of the PV array configuration, selected for the low power simulation are summarized in Table 3, meanwhile the specifications for high power simulation are described in Table 4. 
Table 3. PV array for low power simulation parameters.

\begin{tabular}{cc}
\hline Module & CRM60S125S \\
\hline Maximum power & $57.96 \mathrm{~W}$ \\
Cells per module & 24 \\
Open circuit voltage $\left(V_{o c}\right)$ & $14.5 \mathrm{~V}$ \\
Short-circuit current $\left(I_{s c}\right)$ & $5.51 \mathrm{~A}$ \\
Voltage at MPP $\left(\mathrm{V}_{m p}\right)$ & $11.5 \mathrm{~V}$ \\
Current at MPP $\left(I_{m p}\right)$ & $5.04 \mathrm{~A}$ \\
Temperature coefficient of $V_{o c}$ & $-0.322 \% /{ }^{\circ} \mathrm{C}$ \\
Temperature coefficient of $I_{s c}$ & $0.071996 \% /{ }^{\circ} \mathrm{C}$ \\
Light-generated current $\left(I_{L}\right)$ & $5.5363 \mathrm{~A}$ \\
Diode saturation current $\left(I_{O}\right)$ & $4.6762 \times 10^{-11} \mathrm{~A}$ \\
Diode ideality factor & 0.92399 \\
Shunt resistance $\left(R_{s h}\right)$ & $56.6705 \Omega$ \\
Series resistance $\left(R_{S}\right)$ & $0.25984 \Omega$ \\
Parallel strings & 1 \\
Series-connected strings & 1 \\
\hline
\end{tabular}

Although Tables 3 and 4 summarizes the relevant parameters of each PV array, the parameterization of the dynamic behavior to be optimized can be better characterized by the power and current against voltage curves, since these curves graphically approximate how arrays will behave against different solar irradiances.

Table 4. PV array for high power simulation parameters.

\begin{tabular}{cc}
\hline Module & TP250MBZ \\
\hline Maximum power & $249 \mathrm{~W}$ \\
Cells per module & 60 \\
Open circuit voltage $\left(V_{o c}\right)$ & $36.8 \mathrm{~V}$ \\
Short-circuit current $\left(I_{s c}\right)$ & $8.83 \mathrm{~A}$ \\
Voltage at MPP $\left(\mathrm{V}_{m p}\right)$ & $30 \mathrm{~V}$ \\
Current at MPP $\left(I_{m p}\right)$ & $8.3 \mathrm{~A}$ \\
Temperature coefficient of $V_{o c}$ & $-0.33 \% /{ }^{\circ} \mathrm{C}$ \\
Temperature coefficient of $I_{s c}$ & $0.063805 \% /{ }^{\circ} \mathrm{C}$ \\
Light-generated current $\left(I_{L}\right)$ & $8.8382 \mathrm{~A}$ \\
Diode saturation current $\left(I_{O}\right)$ & $1.0132-10 \mathrm{~A}$ \\
Diode ideality factor & 0.94812 \\
Shunt resistance $\left(R_{s h}\right)$ & $314.7646 \Omega$ \\
Series resistance $\left(R_{s}\right)$ & $0.2914 \Omega$ \\
Parallel strings & 2 \\
Series-connected strings & 10 \\
\hline
\end{tabular}

Thus, Figure 13 shows the current and power curves of the CRM60S125S modules, meanwhile Figure 14 shows the curves for the TP250MBZ modules. In both cases, the black profiles are the characterization of the Current against Voltage response, against different solar irradiation values. Additionally, the blue curves in both plots represents how the Power of the systems reacts against the same irradiance values at a constant $25^{\circ} \mathrm{C}$.

On the power profile curves of the modules (Figures 13 and 14), the Maximum Power Point (MPP) is highlighted by the red circles, where they show how the MPP move regarding different irradiation inputs. Therefore, it is important to highlight that the current and power against voltage profiles were estimated through the model discussed in Section 2, where the estimation of the MPP serves as reference for the simulations in order to validate the results through the expected power solutions. 


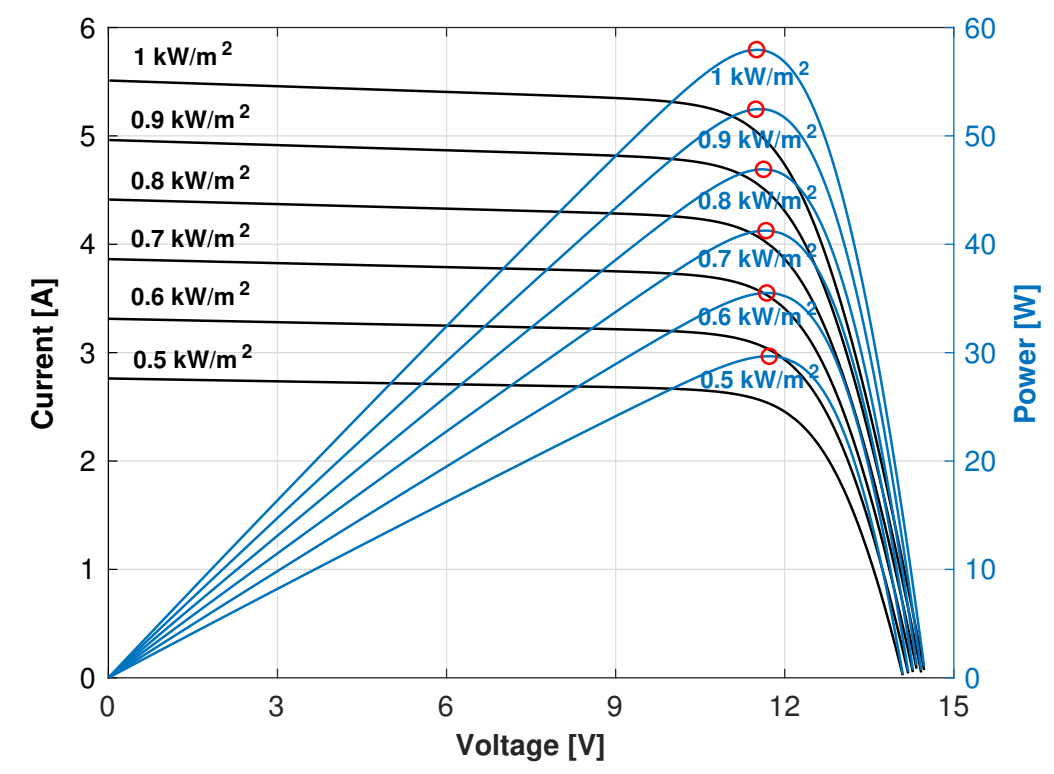

Figure 13. Current (black) and Power (blue) against Voltage curves for low power PV array simulations.

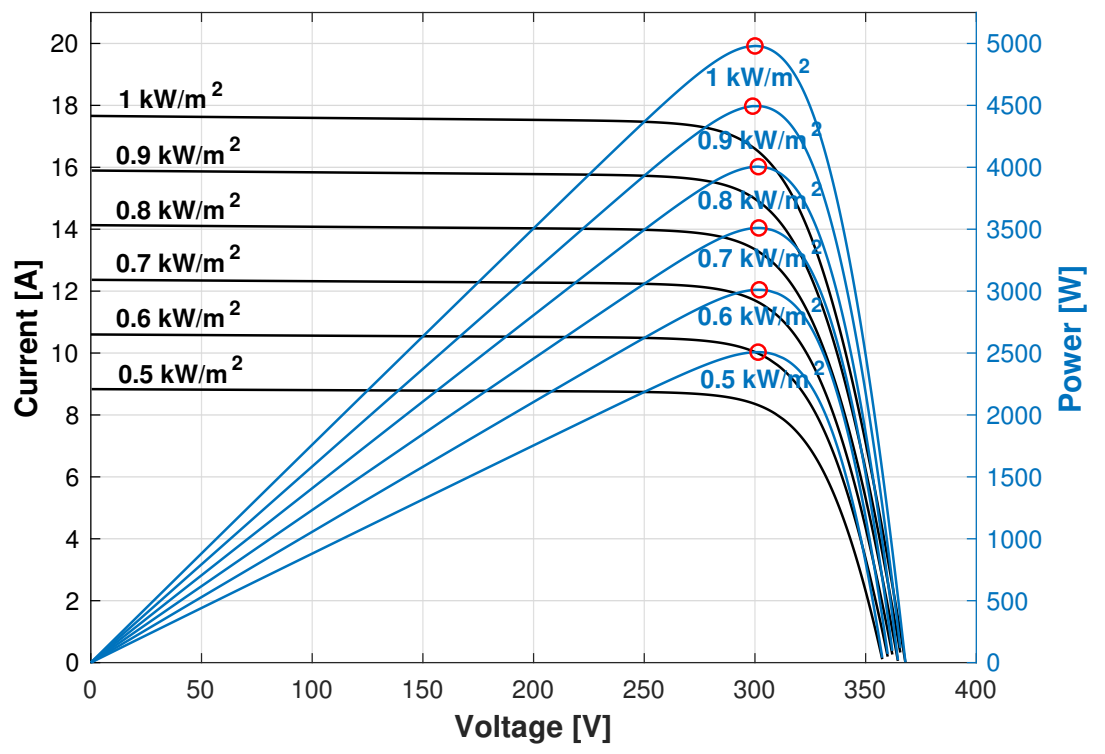

Figure 14. Current (black) and Power (blue) against Voltage curves for high power PV array simulations.

Thus, it was validated that both designed converters were capable of achieving the dynamic optimization, regarding the behavior of the PV arrays shown by Figures 11 and 12, where the power parameters of Figure 11 are in the boundaries of the power capabilities of the converter in Figure 9, and the power profiles from Figure 12 are clearly in the boundaries of the achievable power from the converter in Figure 10.

\section{Results}

The simulations were performed using the model summarized by Figure 8 , where its implementation in the Simulink environment is traduced into the model illustrated in Figure 15 and the set of inputs are taken as the variable irradiation profiles (described in Figures 11 and 12) and the Temperature. On the other hand, the PV source block is taken as the different PV systems described in Tables 3 and 4 . 


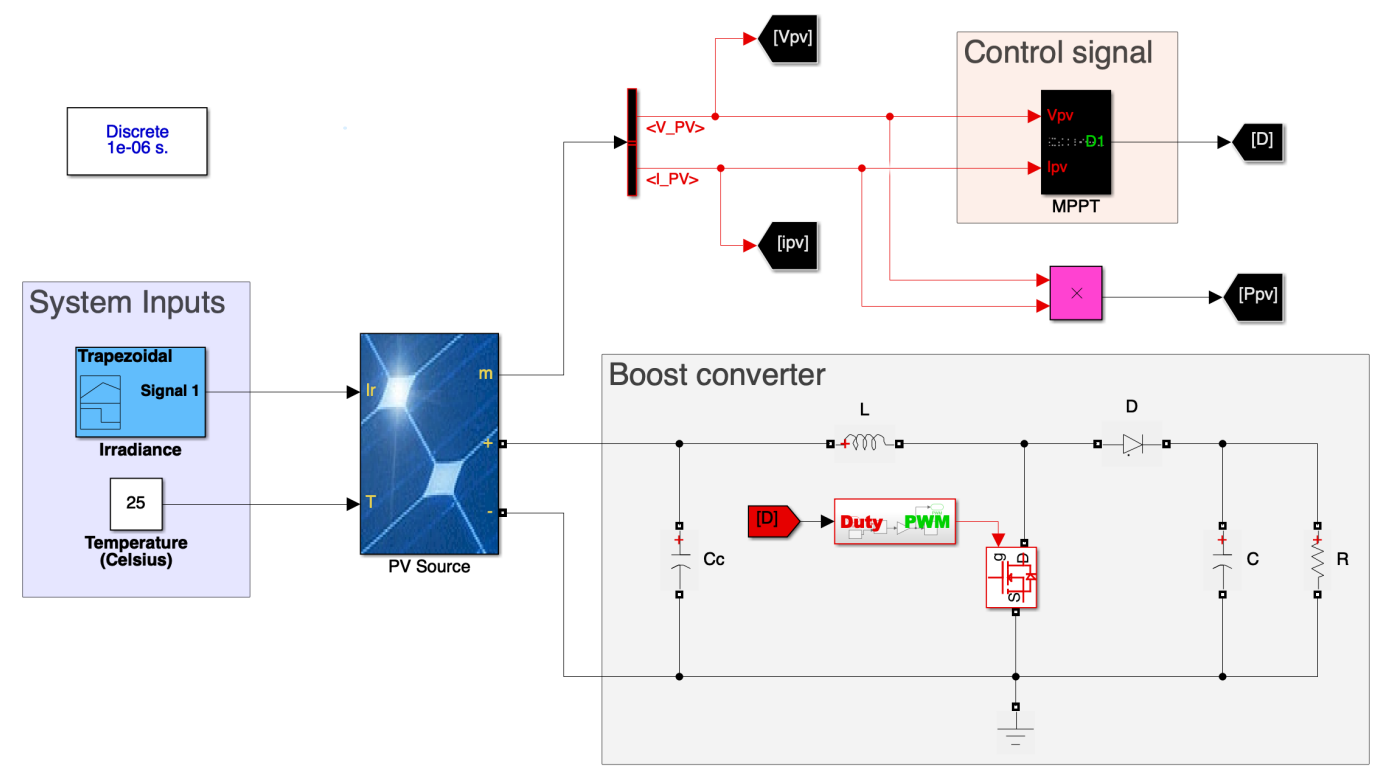

Figure 15. Blocks diagram for the Simulink simulations.

Moreover, the Boost converter is also highlighted in Figure 15, where its parameters change as shown in Tables 1 and 2, regarding the power simulation to be evaluated. Nevertheless, as highlighted in the Boost converter set, the duty cycle is the control parameter obtained from the MPPT algorithms. Finally, it is important to highlight that for the implementation of the PSO- and EA-based algorithms, the obtained output is directly the duty cycle of the system, contrary to what happens with the P\&O implementation, where the duty cycle has to be inverted through the conversion ratio of the DC/DC converter, whereas in this case the duty cycle is taken as $D=1-D$.

Additionally, the MPPT methods were compared through simulations run in MATLAB Simulink, using Simscape Power Systems ${ }^{\mathrm{TM}}$ components. In regards to the dynamic model fidelity capabilities of the software, the tests were performed using the parameters shown in Table 5 where the solver ode4, stands for Ordinary Differential Equations solver 4. Finally, all results in this paper were achieved using a $0.1 \mathrm{~s}$ sample time for all algorithms, meanwhile the EA-based MPPT was implemented using 2 epicenters, and the PSO-based MPPT used a population of 4 particles (which is a particles population also validated in [9]).

Table 5. Simulations parameters used in MATLAB Simulink.

\begin{tabular}{cc}
\hline Simulation mode & Discrete \\
Stop time & $10 \mathrm{~s}$ \\
Sampling time & $1 \mu \mathrm{s}$ \\
Time to start saving data & $0 \mathrm{~s}$ \\
Solver & ode4 (Runge-Kutta) \\
\hline
\end{tabular}

\subsection{Low Power Simulation Results}

Therefore, from the first simulation, it is worth remembering that it was carried out by taking the PV array with the characteristics summarized by Table 3, together with the converter whose parameters are described in Table 1 . Therefore, taking as the expected power behavior the simulated profiles from Figure 13, the tests were performed through the dynamic irradiation input from Figure 11. Thus, Figure 16 shows the Power through time results comparison between the algorithms. 


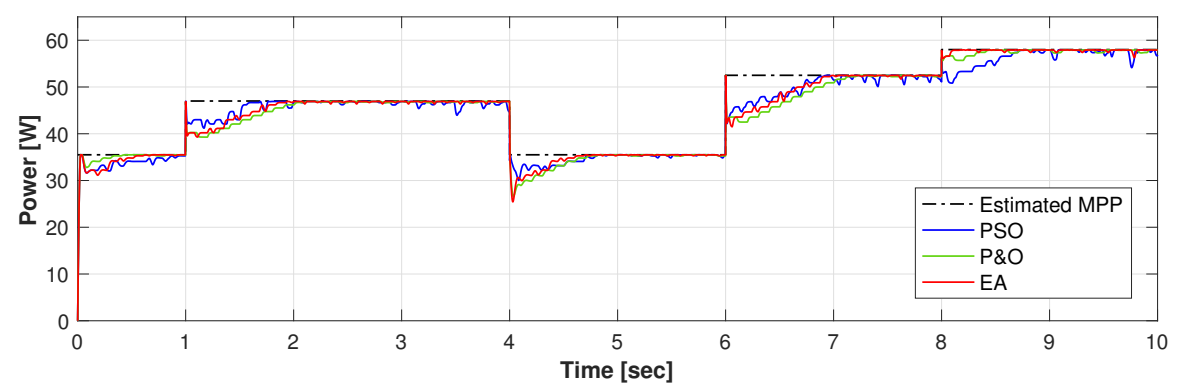

Figure 16. Power harvested through time, regarding the low power simulation.

In all the presented curves in this section, the Red profiles were obtained through the EA, the Blue profiles were obtained by the PSO-based algorithm, the Green ones were obtained from the P\&O results, and finally the segmented and dotted line is the reference estimation obtained through the model discussed in Section 2.

Hence, the $\mathrm{P} \& \mathrm{O}$ algorithm showed its reliability against dynamic changes, always achieving a near MPP approximation, but generally with a slower response regarding the other two algorithms. In spite of the PSO-based algorithm achieving a highly effective response reaching the MPP, there was a highly compromised relation between the performance of the algorithm and the population size (as also validated in [9]).

As shown during the second and third irradiation step, the PSO achieved to find the MPP by gradually reducing the oscillations around it, nevertheless it generally compromised the efficiency when fast dynamic conditions were introduced, shown in the next irradiation changes. Now, regarding the EA-based MPPT, it achieved the fastest recovery in the last three irradiation changes and even an almost immediate solution in the last step, achieving it with lower computational cost regarding the lower epicenters population (compared to the particles population of the PSO).

However, Figures 17 and 18 shows the breakdown of the power obtained, in terms of the voltage and current respectively from the PV source. Where theoretically, as explained in [1], the input voltage in the converter should be nearly constant at a MPP operation mode, the current profile reflects the dynamic behavior of the current.

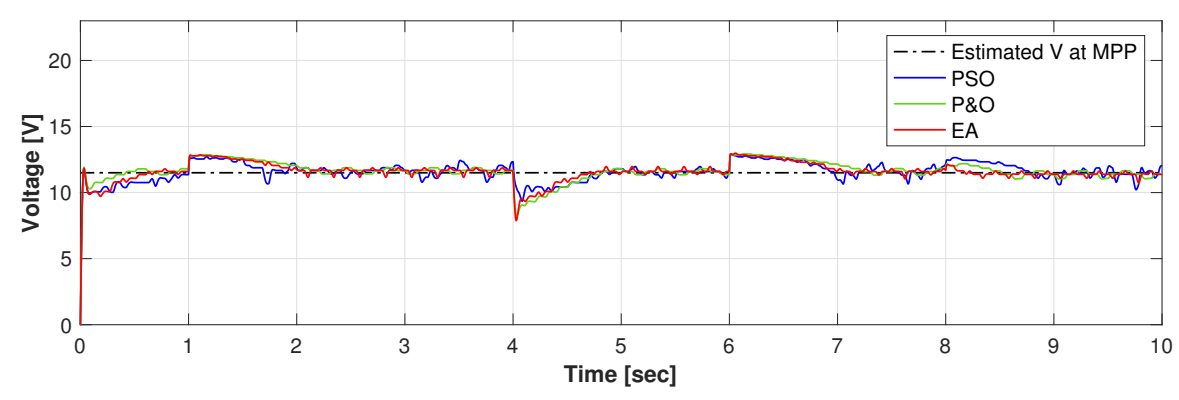

Figure 17. Voltage from the PV source $\left(V_{p v}\right)$ through time from low power simulations.

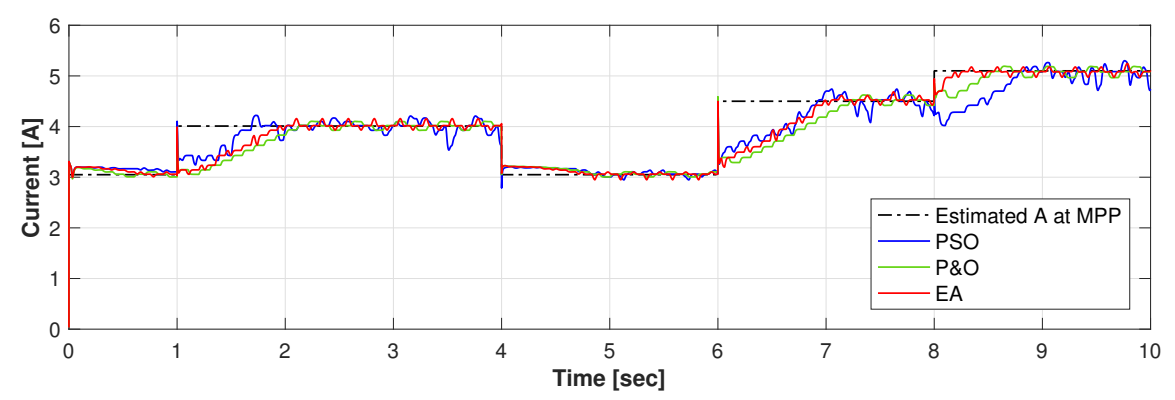

Figure 18. Current from the PV source $\left(i_{p v}\right)$ against time profiles from low power simulations. 
That is the behavior that is shown by the results analyzed through Figures 17 and 18, where on the one hand, the $V_{p v}$ profile showed the dynamic correction of the algorithms when the irradiation changes occurred, achieving a nearly constant value (11.5 V as estimated in Table 3$)$. Nevertheless, the steady voltage state was recovered faster in almost every irradiation change using the EA-based MPPT.

On the other hand, the $i_{p v}$ curve from Figure 18 shows the dynamic behavior of the current through time, where it can be seen how the induced perturbations from the PSO search had a greater effect in the current than on the voltage of the source, and hence responsible for the power oscillations in the $P_{p v}$ performance. Therefore, regarding the current profile, the $\mathrm{P} \& \mathrm{O}$ and the EA solutions provided less $i_{p v}$ ripple, but the EA proved to be a more effective solution due its searching time and the achieved steady-state oscillations.

\subsection{High Power Simulation Results}

Now, speaking of the simulation with greater power, it is important to remember that it was performed by taking the PV array with the characteristics summarized by Table 4, in addition to the converter parameters described in Table 2. In this case, the expected power behavior is the one described in Figure 14. Thus, the irradiation input is taken from Figure 12. Consequently, Figure 19 shows the Power through Time comparison between the algorithms, regarding high power simulation.

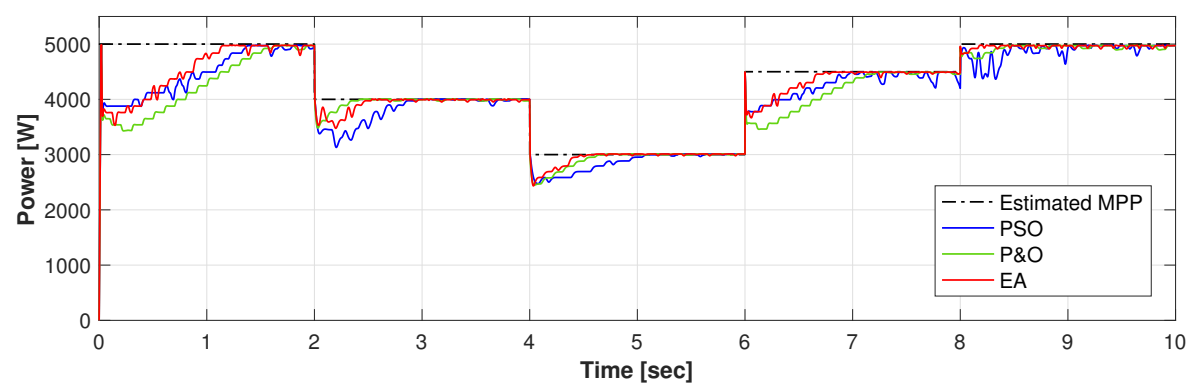

Figure 19. Power harvested through time, regarding high power simulation.

Thereby, after both simulations, the consistency of the results is demonstrated where in the case of the $P_{p v}$ profiles from Figures 16 and 19, the EA-based MPPT showed to be in almost all cases the fastest response. The PSO showed great reliability in always finding the MPP, showing the greatest characteristic of the algorithm, when in all cases it gradually reduced the oscillations around the MPP. Nevertheless, the convergence was not always quickly achieved, showing again a compromised efficiency when fast dynamic conditions were introduced (as also validated in results presented in [9]).

Furthermore, Figures 20 and 21 are the Voltage and Current (respectively) components of the Power curve from Figure 19. Comparing the voltage results from Figures 17 and 20, it can be inferred that in both cases the faster and even smoother response after a parametric deviation, from solar irradiation, was achieved from the EA-MPPT algorithm. Moreover, the $V_{p v}$ maintained an average steady-state against small changes but a quick correction response against greater ones.

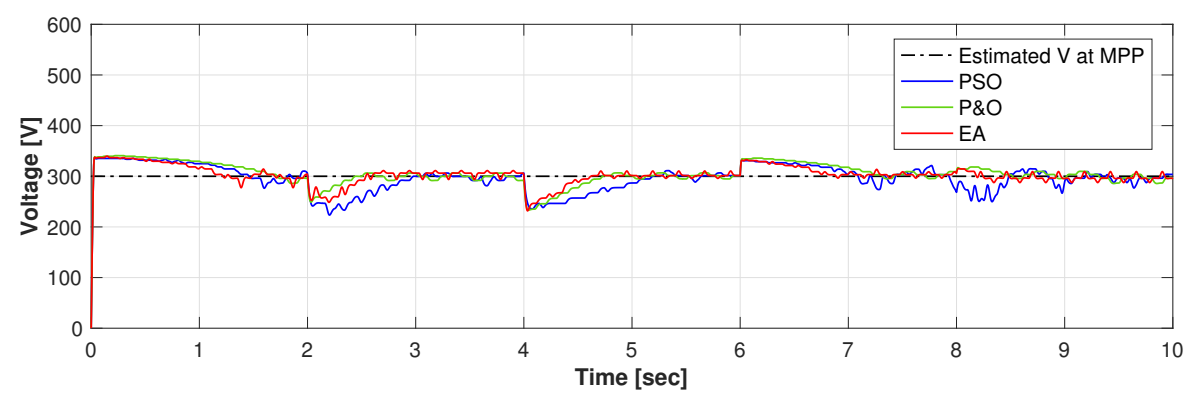

Figure 20. Voltage from the PV source $\left(V_{p v}\right)$ through time from high power simulations. 


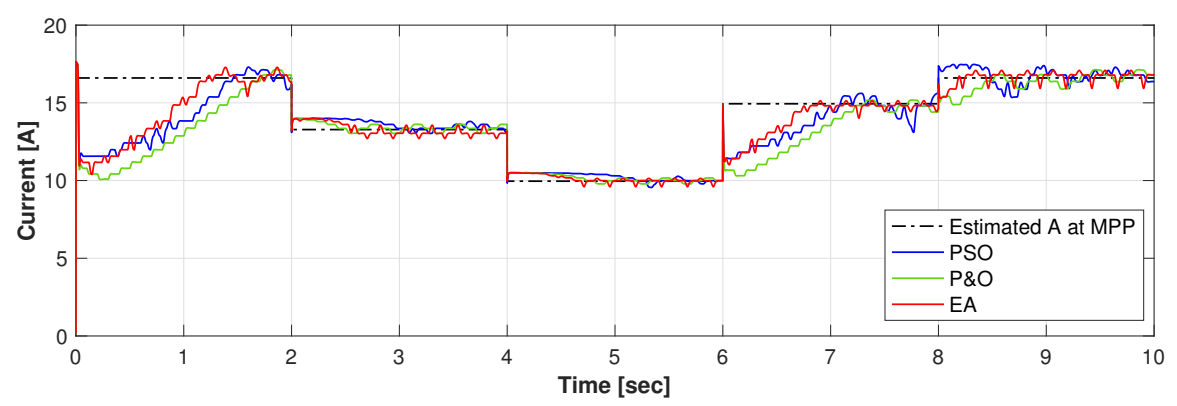

Figure 21. Current from the PV source $\left(i_{p v}\right)$ against time profiles from high power simulations.

Nevertheless, the oscillations induced by the searching behavior of the algorithms could be better analyzed from the $i_{p v}$, where it can be seen that the searching behavior of the PSO algorithm was the most aggressive, regarding its searching performance when fast dynamic changes in the irradiation were induced. Nonetheless, the oscillations were dampened through time, being the main advantage of that approach, in other words, slow changes where the temperature and irradiance inputs remained almost constant, the PSO tended to collect more and more energy due to its convergence capacity.

\subsection{Results Quantification}

Finally, in order to quantify the obtained results, Table 6 summarizes the numerical results from the algorithms where the results are presented for each algorithm in the presented scenarios, reviewing the energy harvested through the simulation time and therefore its efficiency, estimated by contrasting the calculation of the integral of the power curve against the integral of the estimated total power (as detailed in [9]). In all cases from Table 6, the highlighted in red numbers are the best results obtained from the simulations.

For the low power simulation, the estimation of the maximum achievable energy harvested led to be $468.5 \mathrm{~W}$ for the $10 \mathrm{~s}$ simulations, which can be broken down into $40.38 \mathrm{~A}$ as the maximum current to be obtained from the case study. Meanwhile, the achievable power that could be obtained from the higher power simulation resulted in $43.000 \mathrm{~kW}$ for the $10 \mathrm{~s}$ simulations, which can be traduced as 142.7600 A of achievable current through time.

Table 6. Summary of the quantified results.

\begin{tabular}{rcccccc}
\hline & \multicolumn{3}{c}{ Low Power Simulation } & \multicolumn{2}{c}{ High Power Simulation } \\
\cline { 2 - 7 } & P\&O & PSO & EA & P\&O & PSO & EA \\
\hline Energy Harvested [W] & 452.9863 & 453.8238 & 455.6432 & $40.462 \mathrm{k}$ & $40.565 \mathrm{k}$ & $41.388 \mathrm{k}$ \\
MPPT Efficiency [\%] & 96.6886 & 96.8674 & 97.2558 & 94.0977 & 94.3372 & 96.2512 \\
Total Wasted Power [W] & 15.5137 & 14.6762 & 12.8568 & $2.5380 \mathrm{k}$ & $2.4350 \mathrm{k}$ & $1.6120 \mathrm{k}$ \\
Average $V_{p v}$ (Voltage at MPP) [V] & 11.6433 & 11.5714 & 11.5801 & 304.6832 & 295.7527 & 302.1548 \\
Standard Deviation of $V_{M P P}[\mathrm{~V}]$ & 0.7544 & 0.7189 & 0.7179 & 21.2326 & 25.7834 & 18.6481 \\
Current Error in $I_{p v}[\mathrm{~A}]$ & 1.4687 & 1.2222 & 1.0368 & 9.7443 & 5.4588 & 5.6222 \\
\hline
\end{tabular}

Firstly, the indicators regarding the power harvested (Energy Harvested, MPPT Efficiency, and Total Wasted Power), showed a clear trend in the performance of algorithms, where the most efficient algorithm was in both scenarios the EA-based MPPT, consistently achieving the greater power through time, the greatest efficiency, and therefore the lowest wasted energy.

However, regarding the voltage and current components of the energy harvested, the average $V_{p v}$ at MPP showed different results regarding both study cases where the best result regarding the stability reflected by the standard deviation of the voltage signal was achieved in both cases by the EA-MPPT but the result that converged better to the estimated voltage at MPP was obtained by the PSO-MPPT in the lower power simulation (11.5 V at MPP) and by the EA-MPPT in the greater power case study (300 V at MPP). 
On the other hand, the results from the current through time profiles showed that the error through time had its better behavior through the EA-based algorithm in the lower power simulation and through the PSO approach in the higher power case. Nevertheless, it is important to highlight the fact that the best tracking behavior was achieved by both metaheuristic-based approaches where the dynamic optimization issue was dealt better in summary by the proposed optimization algorithm.

Nevertheless, since the PSO- and EA-based algorithms are stochastic optimization methods, the experiments where repeated 35 times in order to acquire a statistical analysis of the energy harvested through time. Therefore, the accuracy histograms from Figures 22 (low power simulations) and 23 (high power simulations), where the accuracy frequency represents the times that the algorithms achieved the presented accuracy percentage from the tracked MPPs.

It is important to highlight that since the $\mathrm{P} \& \mathrm{O}$ algorithm is not stochastic, the accuracy and the energy harvested of the algorithm did not change through the iterations, thus the green bars from the accuracy histograms were only indicating the accuracy of the algorithm obtained in all the performed simulations (99.7735\% and $99.5297 \%$ in the low and high power simulations respectively). Meanwhile, in the case of the $\mathrm{P} \& \mathrm{O}$ algorithm, the energy harvested through all the performed tests was fixed in $452.9863 \mathrm{~W}$ for the low power and $40.462 \mathrm{~kW}$ for high power cases.
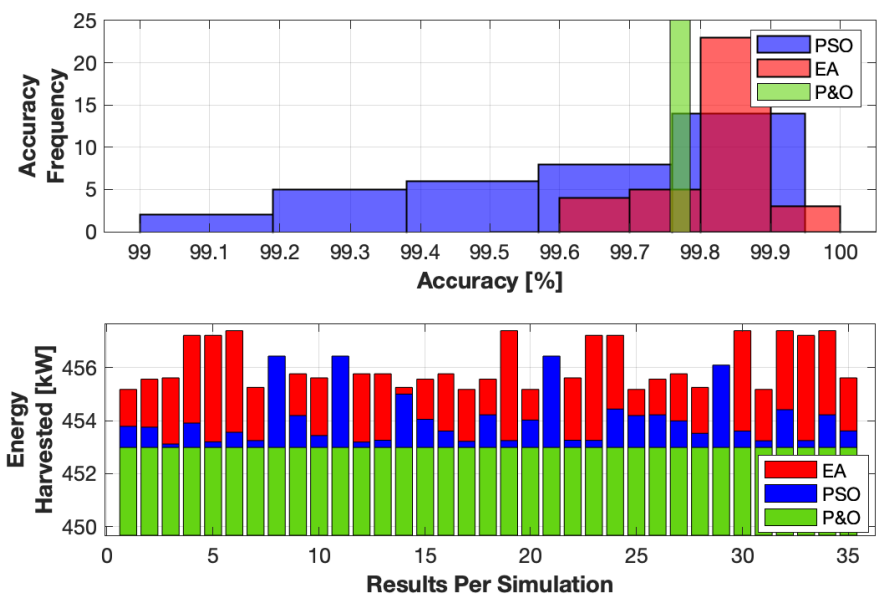

Figure 22. Accuracy of the tracked MPPs and energy harvested per simulation from the low power case study.
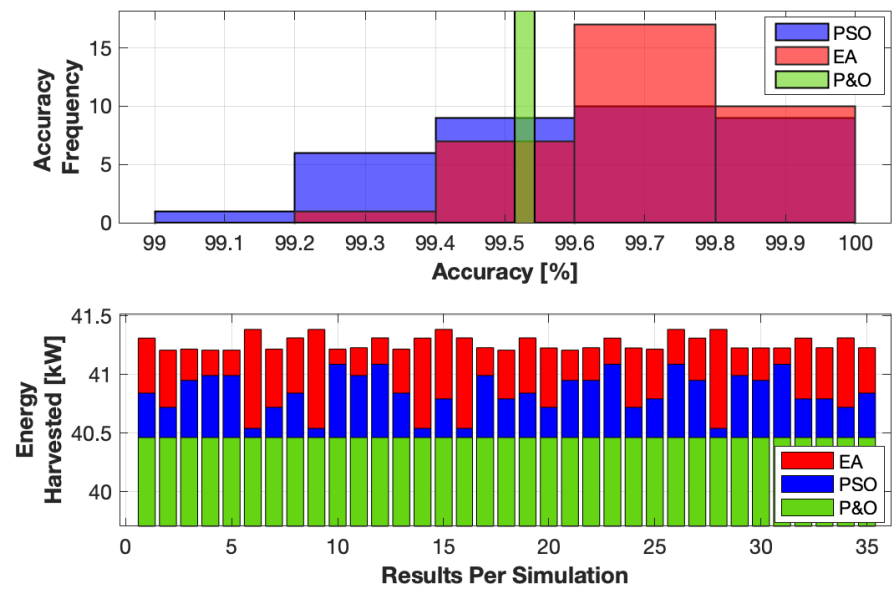

Figure 23. Accuracy of the tracked MPPs and energy harvested per simulation from the high power case study. 
Henceforth, the histograms allow one to validate that statistically, the metaheuristic optimizations trend to perform a more accurate tracking behavior regarding the MPP changed through time yet, the EA-based MPPT showed standard deviation reduction in the low power case, but maintained a higher probability to achieve at least $99.8 \%$ accuracy for either case.

However, the bar plots validated that the energy harvested from the metaheuristic-based algorithms also achieved improved results compared to the P\&O algorithm, where the EA-MPPT achieved the greater energy harvested in all the high power tests, in addition to the performed 31 of the 35 simulated low power tests.

Furthermore, summarizing the results also from Table 6, the implementation of EA-based MPPT found its greatest strengths in high power applications because the greater fluctuations in irradiation were better exploited if the algorithm was more efficient. Evidently, those fluctuations were bigger in higher power applications and therefore it was the greatest opportunity area for this optimization proposal.

Hence, from the quantification of the results, Figure 24 illustrates the performance summary of the algorithms where the calibration dependence regards the complexity of the algorithm calibration for its application. Nonetheless, the scheme from Figure 24 follows a color code referring to three stages: Red stands for the poorest performance, green for the greater one, and the orange level for an intermediate stage between them.

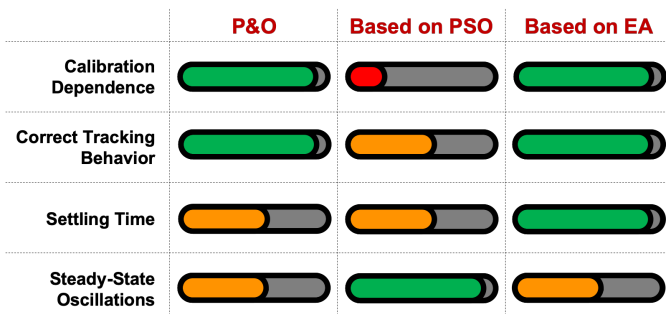

Figure 24. Performance comparison between the analyzed MPPT algorithms.

In the case of the correct tracking behavior, the PSO-based solution was penalized due to its high parameter variation dependence because different population or even algorithm coefficients may drastically affect its performance (as explored in [9]).

Furthermore, the point that contemplates the performance of the algorithms in terms of the settling time evaluates the behavior of the algorithms against the irradiation changes, where the power profiles from Figures 16 and 19 clearly showed the MPPT algorithm based on the EA as the best solution. Nevertheless, the steady-state oscillations of the PSO had no match for scenarios where the irradiation had low dynamic changes in time, which is the best and clearest feature of that solution. Yet, the almost null oscillations in the steady-state from the PSO had an asymptotic behavior with non finite time lapse. In other words, the oscillations were reduced through time (due to the inertia weight from Equation (7)) but with no specific time, which caused uncertainty in the magnitude of the oscillations before dynamic changes by the inputs.

The authors are currently working in the first implementation of the novel MPPT approach in a real PV system. Therefore, future work could contemplate the implementation of the optimization algorithm in an experimental DC/DC converter test bed, with a real irradiation scenario using a PV generator in order to validate the implementability of the algorithm in a real online application. Additionally, the performance analysis of the algorithm against partially shaded conditions is being studied, which is one of the scenarios that are going to be studied in the experimental application.

\section{Conclusions}

From the fact that solar energy finds its greatest strength (compared to other energy sources) in the versatility of implementation in virtually any country in the world, it is also known that no solution 
is able to fully meet all needs. Thus a proposed solution that manages to be an efficient solution in situations where parametric variations in temperature and irradiance is significant. Therefore, the performed tests showed a $17.12 \%$ and a $12.4 \%$ of reduction in the energy wasted in a $10 \mathrm{~s}$ time lapse, compared to the P\&O and PSO-based MPPT respectively. Meanwhile, the proposal achieved to reduce the power wasted in the same $10 \mathrm{~s}$ period, with $33.8 \%$ compared to the PSO solution and $36.48 \%$ compared to the classic $\mathrm{P} \& \mathrm{O}$. These results validated the consistency of the proposal, which achieved in different scenarios a dynamic behavior improvement with an easy suitability between different power designs.

Author Contributions: Conceptualization, E.M., A.O., P.P., and D.B.; Formal analysis, E.M.; Investigation, E.M. and I.M.; Methodology, E.M., A.O., and I.M.; Project administration, A.M.; Resources, A.M.; Software, E.M.; Supervision, P.P., I.M., and A.M.; Validation, A.O. and I.M.; Writing-original draft, E.M.; Writing—review \& editing, P.P. All authors have read and agreed to the published version of the manuscript .

Funding: We appreciate financial support through the Scholarships Program of CONACYT addressed to CVU 859994, Tecnologico de Monterrey.

Conflicts of Interest: The authors declare no conflict of interest.

\section{References}

1. Femia, N.; Petrone, G.; Spagnuolo, G.; Vitelli, M. Power Electronics and Control Techniques for Maximum Energy Harvesting in Photovoltaic Systems; CRC Press: Cleveland, OH, USA, 2017.

2. Dupont, E.; Koppelaar, R.; Jeanmart, H. Global available solar energy under physical and energy return on investment constraints. Appl. Energy 2020, 257, 113968. [CrossRef]

3. IEA. World Energy Outlook 2019; Technical Report; International Energy Agency: Paris, France, 2019.

4. IEA. Renewables 2019. Technical Report; International Energy Agency: Paris, France, 2019.

5. Leva, S.; Ogliari, E. Computational Intelligence in Photovoltaic Systems; MDPI: Basel, Switzerland, 2019

6. Lee, H.S.; Yun, J.J. Advanced MPPT Algorithm for Distributed Photovoltaic Systems. Energies 2019, 12, 3576. [CrossRef]

7. Tan, B.; Ke, X.; Tang, D.; Yin, S. Improved perturb and observation method based on support vector regression. Energies 2019, 12, 1151. [CrossRef]

8. Ishaque, K.; Salam, Z.; Amjad, M.; Mekhilef, S. An improved particle swarm optimization (PSO)-based MPPT for PV with reduced steady-state oscillation. IEEE Trans. Power Electron. 2012, 27, 3627-3638. [CrossRef]

9. Dolara, A.; Grimaccia, F.; Mussetta, M.; Ogliari, E.; Leva, S. An evolutionary-based MPPT algorithm for photovoltaic systems under dynamic partial shading. Appl. Sci. 2018, 8, 558. [CrossRef]

10. Koad, R.B.; Zobaa, A.F.; El-Shahat, A. A novel MPPT algorithm based on particle swarm optimization for photovoltaic systems. IEEE Trans. Sustain. Energy 2016, 8, 468-476. [CrossRef]

11. Eltamaly, A.M.; Al-Saud, M.; Abo-Khalil, A. Performance Improvement of PV Systems' Maximum Power Point Tracker Based on a Scanning PSO Particle Strategy. Sustainability 2020, 12, 1185. [CrossRef]

12. Kermadi, M.; Salam, Z.; Ahmed, J.; Berkouk, E.M. An effective hybrid maximum power point tracker of photovoltaic arrays for complex partial shading conditions. IEEE Trans. Ind. Electron. 2018, 66, 6990-7000. [CrossRef]

13. Mendez, E.; Ortiz, A.; Ponce, P.; Acosta, J.; Molina, A. Mobile Phone Usage Detection by ANN Trained with a Metaheuristic Algorithm. Sensors 2019, 19, 3110. [CrossRef]

14. Ponce-Cruz, P.; Molina, A.; Ramírez-Mendoza, R.; Mendez-Flores, E.; Ortiz, A.; Balderas, D. A Practical Approach to Metaheuristics using LabVIEW and MATLAB ${ }^{\circledR}$; Chapman and Hall/CRC: Oxfordshire, UK, 2020.

15. Tascioni, R.; Cioccolanti, L.; Del Zotto, L.; Habib, E. Numerical Investigation of Pipelines Modeling in Small-Scale Concentrated Solar Combined Heat and Power Plants. Energies 2020, 13, 429. [CrossRef]

16. Agbemuko, A.J.; Domínguez-García, J.L.; Prieto-Araujo, E.; Gomis-Bellmunt, O. Impedance modelling and parametric sensitivity of a VSC-HVDC system: New insights on resonances and interactions. Energies 2018, 11, 845. [CrossRef]

17. Alnuman, H.; Gladwin, D.; Foster, M. Electrical modelling of a DC railway system with multiple trains. Energies 2018, 11, 3211. [CrossRef] 
18. Tseng, S.Y.; Wang, H.Y. A photovoltaic power system using a high step-up converter for DC load applications. Energies 2013, 6, 1068-1100. [CrossRef]

19. Krommydas, K.F.; Alexandridis, A.T. Power controller design and stability analysis of a photovoltaic system with a dc/dc boost converter. In Proceedings of the 52nd IEEE Conference on Decision and Control, Florence, Italy, 10-13 December 2013; pp. 3629-3634.

20. Dalala, Z.M.; Saadeh, O.; Bdour, M.; Zahid, Z.U. A new maximum power point tracking (MPPT) algorithm for thermoelectric generators with reduced voltage sensors count control. Energies 2018, 11, 1826. [CrossRef]

21. Rivera, J.; Ortega-Cisneros, S.; Chavira, F. Sliding Mode Output Regulation for a Boost Power Converter. Energies 2019, 12, 879. [CrossRef]

22. Krommydas, K.F.; Alexandridis, A.T. Design and passivity-based stability analysis of a PI current-mode controller for dc/dc boost converters. In Proceedings of the 2014 American Control Conference, Portland, OR, USA, 4-6 June 2014; pp. 5067-5072.

23. Erickson, R.W.; Maksimovic, D. Fundamentals of Power Electronics; Springer Science \& Business Media: Berlin, Germany, 2007.

24. Bellia, H.; Youcef, R.; Fatima, M. A detailed modeling of photovoltaic module using MATLAB. NRIAG J. Astron. Geophys. 2014, 3, 53-61. [CrossRef]

25. Xiong, G.; Zhang, J.; Yuan, X.; Shi, D.; He, Y. Application of symbiotic organisms search algorithm for parameter extraction of solar cell models. Appl. Sci. 2018, 8, 2155. [CrossRef]

26. Louzazni, M.; Khouya, A.; Amechnoue, K.; Gandelli, A.; Mussetta, M.; Crăciunescu, A. Metaheuristic algorithm for photovoltaic parameters: comparative study and prediction with a firefly algorithm. Appl. Sci. 2018, 8, 339. [CrossRef]

27. Petrone, G.; Luna, M.; La Tona, G.; Di Piazza, M.; Spagnuolo, G. Online Identification of Photovoltaic Source Parameters by Using a Genetic Algorithm. Appl. Sci. 2018, 8, 9. [CrossRef]

28. Eberhart, R.; Kennedy, J. A new optimizer using particle swarm theory. MHS'95. In Proceedings of the Sixth International Symposium on Micro Machine and Human Science, Nagoya, Japan, 4-6 October 1995; pp. 39-43.

29. Solihin, M.I.; Tack, L.F.; Kean, M.L. Tuning of PID controller using particle swarm optimization (PSO). Int. J. Adv. Sci. Eng. Inf. Technol. 2011, 1, 458-461. [CrossRef]

30. Jali, M.; Azam, M.; Ghazali, R.; Jaafar, H.; Bohari, Z. PID control tuning using PSO for prosthetic hand system. Int. J. Appl. Eng. Res. 2016, 11, 10274-10280.

31. Clerc, M. The swarm and the queen: towards a deterministic and adaptive particle swarm optimization. In Proceedings of the 1999 Congress on Evolutionary Computation-CEC99 (Cat. No. 99TH8406), Washington, DC, USA, 6-9 July 1999; Volume 3, pp. 1951-1957.

32. Ponce, P.; Molina, A. Earthquake Optimization Method: An Internal Research Report during the Project; Tecnologico de Monterrey; Binational Laboratory: Mexico City, Mexio, 2018.

33. Mendez, E.; Ortiz, A.; Ponce, P.; Molina, A. Electric machines control optimization by a novel geo-inspired earthquake metaheuristic algorithm. In Proceedings of the 2018 Nanotechnology for Instrumentation and Measurement (NANOfIM), Mexico City, Mexio, 7-8 November 2018; pp. 1-6.

34. Dayaramani, R.; Bharadwaj, S.; Gawre, S. Simulation and Designing of MPPT Based Solar PV System with DC-DC Boost Converter. ISA Trans. 2017, 4, 562-576.

35. Hayat, A.; Faisal, A.; Javed, M.Y.; Hasseb, M.; Rana, R.A. Effects of input capacitor (cin) of boost converter for photovoltaic system. In Proceedings of the 2016 International Conference on Computing, Electronic and Electrical Engineering (ICE Cube), Baluchistan, Pakistan, 11-12 April 2016; pp. 68-73.

(C) 2020 by the authors. Licensee MDPI, Basel, Switzerland. This article is an open access article distributed under the terms and conditions of the Creative Commons Attribution (CC BY) license (http:// creativecommons.org/licenses/by/4.0/). 